\title{
Abstracts of the 2014 Annual Meeting of the AANS/CNS Section on Disorders of the Spine and Peripheral Nerves
}

\author{
Orlando, Florida • March 5-8, 2014 \\ (DOI: 10.3171/2014.3.FOC-DSPNabstracts)
}

\section{MAYFIELD BASIC SCIENCE}

100. Cerebrospinal Fluid Drainage and Induced Hypertension Improve spinal cord Perfusion in the Setting of Acute Spinal Cord Injury

Nikolay L. Martirosyan MD (University of Arizona), M. Yashar S. Kalani BS MS MD PhD (Barrow Neurological Institute), William Bichard, Ali A. Baaj MD (University of Arizona), L. Fernando Gonzalez MD, Robert F. Spetzler MD (Barrow Neurological Institute), Mark C. Preul MD (Barrow Neurological Institute), Nicholas Theodore M.D. F.A.C.S. (Barrow Neurosurgical Associates)

Introduction: Elevation of mean arterial blood pressure (MAP) and cerebrospinal fluid drainage (CSFD) has been used as treatment modalities in patients after acute spinal cord injury (SCI). Currently there is no evidence in support of the routine use of either modality. We sought to determine efficacy of aggressive MAP augmentation combined with lowering of intrathecal pressure (IP) by CSFD to improve spinal cord blood flow (SCBF) after SCI.

Methods: We induced mild spinal cord injury at the T5 level in pigs. The animals were divided evenly between five groups: Control (laminectomy) $(n=3)$; SCI only $(n=3)$; SCI with elevated MAP (SCI+MAP) $(n=3)$; SCI with CSFD (SCI+CSFD) $(n=3)$; SCI with CSFD and elevated MAP (SCI+MAP+CSFD) $(n=3)$. Elevated MAP and CSFD was initiated 1 hour after SCI. CSF diversion was achieved via lumbar drain. Elevated MAP was achieved by continuous injection of Phenylephrine. Parameters before SCI, at 1h, $2 \mathrm{~h}, 3 \mathrm{~h}$ and $4 \mathrm{~h}$ after SCI were analyzed. All animals underwent continuous monitoring and recording of IP, SCBF at SCI level and MAP.

Results: The SCBF in SCI group was decreased by $56 \%$ after $\mathrm{SCI}$ in comparison with baseline. Increase in blood pressure after SCI resulted in a $34 \%$ decrease in SCBF, whereas CSFD resulted in a 59\% decrease in SCBF. The combination of CSFD and induced hypertension resulted in a $24 \%$ increase in SCBF. The SCI group had stable IP throughout experiment. The SCI+MAP group had an average of $5.45 \mathrm{mmHg}$ IP increase after MAP increase 1 hour after SCI.

Conclusions: SCI does not result in increase in IP. Increased MAP in the setting of SCI causes increase in IP, which results in decreased spinal cord perfusion pressure (SCPP) (SCPP=MAP-IP). Both elevated MAP and CSFD showed only short-term improvement of SCBF followed by hypoperfusion when implemented independently. The combination of increased MAP and CSFD improves SCBF after SCI.

\section{OUTCOMES AWARD}

101. Outcomes After ALIF versus TLIF For Treatment of Symptomatic L5-S1 Spondylolisthesis: A Prospective, MultiInstitutional Comparative Effectiveness Study

Owoicho Adogwa M.D. M.P.H (Duke University Medical Center, Division of Neurosurgery), Tiffany Hodges, Paul Thompson BS (Division of Neurosurgery), Parastou Fatemi, Joel Martin, Timothy Darlington, keshov Sharma, Max Ostrinsky Krucoff MD (Duke Univsrsity Hospital), Joseph S. Cheng MD MS (Vanderbilt University Medical Center), Robert E. Isaacs MD (Duke University Medical Center)

Introduction: Anterior lumbar interbody fusion (ALIF) and transforaminal lumbar interbody fusion (TLIF) procedures achieve lumbar interbody arthrodesis. No recent study has compared improvement in pain and functional disability after both procedures. Whether ALIF or TLIF for treatment of symptomatic L5-S1 spondylolisthesis results in superior post-operative functional outcomes remains unknown. The primary aim of the study was to compare post-operative functional outcomes and complication rates following both surgical approaches.

Methods: A nationwide, multi-institutional, prospective spine outcomes registry was utilized for this study. Enrollment criteria included available demographic, surgical and clinical outcome data. The study included 519 patients with degenerative disc disease or spondylolisthesis at L5-S1; 219 patients underwent ALIF and 300 patients underwent TLIF. Patients completed the Oswestry Disability Index (ODI), MOS Short Form 36 (SF-36), and back and leg pain numerical rating scores before surgery, then at $3,6,12$, and 24 months after surgery. Clinical outcomes and complication rates were compared between both patient cohorts.

Results: Patients undergoing TLIF experienced higher rates of post-operative complication (ALIF: $12.3 \%$, TLIF: $7.8 \%, \mathrm{p}=0.03$ ); however, the likelihood of visceral/vascular injury was significantly higher in the ALIF cohort $(\mathrm{p}=0.002)$. At two years, both ALIF and TLIF patients showed similar 2-year improvement in VAS for back pain (ALIF: $2.98 \pm 3.69$, TLIF: $2.51 \pm 3.73, p=0.15$ ) and leg pain (ALIF: $2.96 \pm 4.09$, TLIF: $2.75 \pm 4.32, \mathrm{p}=0.57$ ). ODI (ALIF: $14.63 \pm 20.36$, TLIF: $14.13 \pm 22.45, \mathrm{p}=0.79$ ), and SF-36 PCS (ALIF: $6.45 \pm 14.15$, TLIF: $7.88 \pm 17.35, \mathrm{p}=0.30$ ) and SF-36 MCS (ALIF: $5.18 \pm 20.92$, TLIF: $5.24 \pm 22.28, \mathrm{p}=0.97$ ) were also improved. Notably, patients undergoing ALIF appear to have a more rapid reduction in one-year VAS back and leg pain scores.

Conclusions: For patients with symptomatic L5-S1 spondylolisthesis, ALIF and TLIF have comparable long-term improvement on pain and functional disability. While ALIF was associated with lower post-operative complication rates and more rapid reduction in one-year back pain, leg pain and functional disability, patients undergoing ALIF were more likely to experience intra-operative visceral or vascular injury.

102. Pre-operative Depression, ODI Scores, and EQ-5D Scores are Significant Factors in Patient Satisfaction Following Lumbar Spine Surgery

Timothy C. Ryken MD MS FACS (Iowa Brain \& Spine Institute), Laura Chapin PhD, Ward Kelly RT(R)

Introduction: Objectives of health care reform highlight reducing costs and improving patient outcomes. Of particular interest are spinal surgeries, often performed on chronic back pain patients unresponsive to non-operative care. The patient experience is important and we investigate which factors predict overall patient satisfaction following surgery. The objective of this study is to examine the relationships between patient satisfaction and comorbidities to determine successful candidates for lumbar surgery.

Methods: A total of 96 patients who underwent lumbar spine surgeries at Covenant Medical Center and were included in the National Neurosurgery Quality and Outcomes Database were assessed pre-operatively, and at 3 (96 patients) and 12 months (31 patients) post-operatively using self-assessment tools. The patient's overall level of satisfaction ODI and EuroQol-5D scores were compiled. Comorbidities including diabetes, smoking, and depression were examined to identify factors predicting patient satisfaction 


\section{Meeting Abstracts: AANS/CNS Section on Disorders of the Spine and Peripheral Nerves}

post-spinal surgery. Vendors of implants and bone grafts were included as variables.

Results: The frequency of post-operative patient satisfaction was compared to pre-operative comorbidities including depression, smoking, diabetes, obesity (BMI $>30$ ), morbid obesity (BMI $>40)$, and vendor of implants and bone grafts. The only comorbidity that was statistically linked with patient satisfaction at 3 months following surgery was depression, with nearly $62 \%$ of dissatisfied patients and only $35 \%$ of satisfied patients identifying themselves as having "Depression Disorder" pre-operatively. We found that patients having worse ODI and EQ-5D scores showed greater improvements in their scores at 3 months following surgery, with statistically significant correlative relationships. Additionally, ODI scores at 3 months were not significantly different than scores at 12 months, suggesting 3 month ODI scores can predict longer-term patientreported outcomes.

Conclusions: Pre-operative depression, ODI and EQ-5D scores are significantly associated with patient satisfaction at 3 months post-surgery and patient satisfaction remains stable comparing 3 and 12-month results.

103. Two Year Prospective, Multicenter Analysis of Consecutive Adult Spinal Deformity (ASD) Patients Demonstrates Higher Fusion Grade, Lower Implant Failures and Greater Improvement in SRS-22r Scores for Patients Treated with Recombinant Human Bone Morpho

Kai-Ming G. Fu MD PhD (Weill Cornell Medical College), Eric Klineberg MD (University of California - Davis), Shay Bess MD (Rocky Mountain Scoliosis and Spine Center), Breton G. Line BSME (Rocky Mountain Scoliosis \& Spine), Virginie Lafage PhD (NYU Langone Medical Center), Frank Schwab MD, PhD, Christopher P. Ames MD (University of California San Francisco Neuro Surgery), Oheneba Boachie-Adjei MD, Douglas C. Burton MD, Khaled Kebaish MD, Robert Hart MD (Ohsu Spine Center), Gregory Mundis MD (San Diego Center for Spinal Disorders), Richard A. Hostin MD, Justin S. Smith MD PhD (University of Virginia Health System), Christopher I. Shaffrey MD FACS (University of Virginia), International Spine Study Group

Introduction: Theoretical advantages of BMP use include high fusion rates and improved outcomes, however little data exists evaluating fusion grade, complications and health related quality of life (HRQOL) for ASD patients treated with BMP. Purpose: evaluate fusion grade, complications and HRQOL associated with BMP vs. no BMP use in a prospective, multicenter, consecutive ASD cohort, minimum 2 year follow-up.

Methods: Multicenter, prospective analysis of consecutive ASD patients receiving BMP (BMP) or no BMP (NOBMP). Inclusion criteria: $\mathrm{ASD}$, age $=18$ years, spinal fusion $=4$ levels, complete demographic and radiographic data, and minimum two-year follow up. $\mathrm{ASD}=\mathrm{scoliosis}=20$ degrees, sagittal vertical axis $=5 \mathrm{~cm}$, pelvic tilt $=25$ degrees, or thoracic kyphosis $>60$ degrees. Spine fusion evaluated using Lenke grade, complications noted, baseline and 2 year postoperative HRQOL (SRS-22r, SF-36, ODI) analyzed.

Results: 141 of 189 patients had complete two year data (75\% follow-up); mean follow up 35.8 months (range 24.1-47.9). BMP $(\mathrm{n}=110$; mean BMP doses: posterolateral $=2.6 \mathrm{mg} / \mathrm{level}$, interbody $=$ $5.3 \mathrm{mg} /$ level $)$ and NOBMP $(\mathrm{n}=31)$ had similar preop deformity, baseline HRQOL, and total posterior fusion levels (BMP $=11.6$, NOBMP=12.9). BMP was older (56 vs. 49 years), had more anteroposterior surgery ( 25 vs. $6.5 \%$ ), and fewer pedicle subtraction osteotomy/patient (0.12 vs. 0.3$)$, than NOBMP, respectively $(\mathrm{p}<0.05)$. BMP had more minor complications (61\% vs. $29 \%)$ and fewer implant failures (1.8 vs. 13\%) than NOBMP, respectively $(\mathrm{p}<0.05)$. Mean BMP fusion grade was greater than NOBMP $(1.9$ vs. 1.5; $\mathrm{p}<0.05)$. BMP had greater 2 year improvement in SRS-22r total (0.9 vs. 0.5$)$, mental ( 0.4 vs. -.02$)$, and pain (1.0 vs. 0.4$)$ scores than NOBMP, respectively $(\mathrm{p}<0.05)$.
Conclusions: BMP use in ASD, at reported BMP dose/level, demonstrated higher fusion grade, fewer implant failures, similar major complications, and greater HRQOL improvement at 2 year follow-up than NOBMP. Research is needed evaluating long term complications and outcomes.

\section{Punctured Intervertebral Discs}

Peter Grunert MD (Weill Cornell Brai and Spine Center), Brandon Borde (Cornell University Department of Biomedical Engineering), Sara Towne (Weill Cornell Medical College), Lawrence J Bonassar PhD, Roger Hartl MD (New York Hospital/Cornell Medical Center)

Introduction: Open defects compromise the ability of the annulus fibrosus (AF) to contain nuclear tissue in the disc space, and therefore increase the likelihood of reherniation. $[1,2]$ We evaluated whether high-density collagen (HDC) gel can prevent nuclear tissue extrusion and subsequent degenerative changes to intervertebral discs in a rat tail spine.

Methods: Forty athymic rats were punctured with an 18-gauge needle at $\mathrm{C} 3 / 4$ in the caudal spine. Uncrosslinked high-density collagen (HDC) $(n=6)$ or HDC cross-linked with $0.25 \mathrm{mM}(\mathrm{n}=9)$, $0.5 \mathrm{mM}(\mathrm{n}=8)$, or $0.75 \mathrm{mM}(\mathrm{n}=8)$ Riboflavin $(\mathrm{RF})$ was injected into the puncture defect of 31 rats. Nine rats were punctured and left untreated. 7T MRIs were obtained at 1, 2,5,12, and 18 week time points to evaluate NP size and degeneration grade. We developed an algorithm based on T2 Relaxation Time measurements to assess NP size by number of MRI voxels that composed it. Lateral X-rays were taken to measure disc heights. Histological analyses were performed to study degenerative changes, and repair of annular defects. Stressrelaxation and frequency sweep tests measured ability of punctured discs to pressurize and their damping quality.

Results: After 18 weeks, untreated discs showed signs of terminal degenerative changes on MRI, histological sections, and biomechanical tests. No NP tissue remained in the disc space. In contrast, discs treated with 0.5 and $0.75 \mathrm{mM}$ cross-linked collagen retained $70 \%$ of NP and $80 \%$ of disc height with minimal degenerative changes on histological section. Biomechanical tests revealed similar damping and pressurization qualities as in healthy discs. Increased RF concentration significantly correlated with NP size and disc height. On histological section, discs treated with crosslinked collagen showed formation of a fibrous cap which repaired the defect. This fibrous cap was formed from host fibroblasts which infiltrated and reorganized injected collagen gel.

Conclusions: HDC can repair annular defects in rat IVDs and maintain their biomechanical properties.

105. Adjacent Segment Degeneration Incidence Reduced after Total Disc Replacement Compared with Anterior Discectomy and Fusion for Treatment of One and Two Level Cervical Degenerative Disc Disease: 4-Year Results from an FDA IDE Clinical Trial

David A. Cavanaugh MD (Louisiana Spine), Pierce D. Nunley MD, Eubulus J. Kerr MD, Andrew Utter MD (Louisiana Spine), Marcus B. Stone PhD (Spine Institute of Louisiana)

Introduction: Maintaining motion at the treated segment to reduce adjacent segment degeneration is a primary reason for the development of recently approved total disc arthroplasty (TDR) devices. Long-term data comparing these devices with ACDF is limited. The purpose of this study is to compare the effect of one and two-level ACDF to one and two-level TDR on the integrity of adjacent segments.

Methods: Patients (575 total; 1 level = 164 TDR, 81 ACDF; 2 level $=225 \mathrm{TDR}, 105 \mathrm{ACDF}$ ) included in this study were participating in the FDA IDE clinical trial of the Mobi-C Cervical Artificial 


\section{Meeting Abstracts: AANS/CNS Section on Disorders of the Spine and Peripheral Nerves}

Disc (LDR Medical; Troyes, France) conducted at 24 investigational sites in the US. All patients were diagnosed with symptomatic DDD at one level or two contiguous levels from $\mathrm{C} 3 / \mathrm{C} 4$ to $\mathrm{C} 6 / \mathrm{C} 7$. Independent radiologists (MMI Inc., Houston, TX) evaluated adjacent intervertebral segments at preoperative baseline and 12, 24, 36, and 48 months post-operation according to the Kellgren-Lawrence (K-L) scale. Any worsening of K-L score from pre-operative assessment to post-operative assessment was considered adjacent level degeneration.

Results: Degenerative changes were observed across all groups and time points. However, for one-level cases, radiologists observed significantly fewer degenerative changes in the TDR group at the superior adjacent segment at 48 months and at the inferior adjacent segment at 12, 24, 36, and 48 months. The two-level TDR group had significantly fewer patients with radiographically identified degeneration of both the superior and inferior adjacent segments at $12,24,36$, and 48 months compared to ACDF.

Conclusions: The rate of adjacent segment degeneration is less in TDR when compared with ACDF. The preservation of segmental range of motion after TDR may result in less symptomatic adjacent level degeneration over time, but further analysis will be necessary to determine the relationship between adjacent level degeneration and disease.

106. Less Invasive Surgery for Treating Adult Spinal Deformities (ASD): Ceiling Effects for Cobb Angle Correction with Three Different Techniques

Kai-Ming G. Fu MD PhD (Weill Cornell Medical College), Michael $Y$. Wang MD FACS (University of Miami), Praveen V. Mummaneni $M D$ (University of California San Francisco Spine Center), Neel Anand MD, David O. Okonkwo MD PhD (University of Pittsburgh), Adam S. Kanter MD (University of Pittsburgh Medical Center Presbyterian), Frank La Marca MD (University of Michigan), Paul Park MD (University Of Michigan, Department of Neurosurgery), Richard G. Fessler MD (Rush University Medical Center), Juan S. Uribe MD (University of South Florida - Division of Neurosurgery), Christopher I. Shaffrey MD FACS (University of Virginia), Virginie Lafage PhD (NYU Langone Medical Center), Raqeeb M. Haque MD (Cleveland Clinic Foundation), Vedat Deviren MD (Univeristy of California San Francisco), Gregory Mundis MD (San Diego Center for Spinal Disorders), International Spine Study Group

Introduction: Minimally invasive surgery (MIS), including transpsoas lateral interbody fusion (LLIF), transforaminal interbody fusion (TLIF), and percutaneous pedicle screw instrumentation (PPI) are being increasingly applied to treat ASD. This study was undertaken to evaluate current limitations of MIS approaches for ASD.

Methods: A retrospective review of multicenter prospectively collected data of 85 consecutive patients with ASD undergoing MIS surgery. Inclusion criteria: age $>45 \mathrm{yrs}$; minimum $20^{\circ}$ coronal lumbar Cobb; 1 year follow up. Procedures were classified as: 1) Standalone (saMIS): MIS LIF only (N=7); 2) cMIS: LIF or MIS TLIF with PPI (N=43); or 3) Hybrid (HYB) - LIF with open posterior instrumented fusion $(\mathrm{N}=35)$.

Results: An average of 4.2 discs (range 2-7) were fused with mean follow-up of 26.1 months. saMIS: mean Cobb changed $34.3^{\circ}$ to $29.5^{\circ}(\mathrm{p}>0.05)$. Cobb range was $23^{\circ}$ to $81.5^{\circ}$, with $57 \%(\mathrm{n}=4)$ greater than $30^{\circ}$ and $28.6 \%(\mathrm{n}=2)$ greater than $50^{\circ}$. A ceiling effect of $23^{\circ}$ for curve correction was observed, regardless of preoperative curve severity.cMIS: mean preop Cobb was $31.1^{\circ}\left(18.6^{\circ}-62^{\circ}\right)$, corrected to $10.3^{\circ}(\mathrm{p}<0.05) .44 \%(19 / 43)$ had a coronal curve greater than $30^{\circ}$ and $4.7 \%(2 / 43)$ greater than $50^{\circ}$. Coronal corrections ranged from $9^{\circ}$ to $34^{\circ}$. A ceiling effect of $34^{\circ}$ for curve correction was observed. HYB: mean preop Cobb was $42.4^{\circ}\left(23^{\circ}-81.5^{\circ}\right)$, corrected to $14.9^{\circ}(\mathrm{p}<0.05) .74 \%(26 / 35)$ had a coronal curve greater than $30^{\circ}$ and $23 \%(8 / 35)$ greater than $50^{\circ}$. Coronal corrections ranged from $4^{\circ}$ to $61^{\circ}$. A ceiling effect of $55^{\circ}$ for curve correction was observed.

Conclusions: The ability to correct a scoliotic curve was directly related to the surgical technique utilized in this series. Our data indicate that MIS platforms for curve correction are currently limited based upon their ceiling effects. Surgeons employing MIS techniques should use these limitations in preoperative planning.

107. HRQoL Scores and Radiographic Parameters Do Not Drive Patient Satisfaction after Adult Spinal Deformity Surgery

D. Kojo Hamilton MD (Oregon Health \& Sciences University), Jayme Hiratzka MD, Shay Bess MD (Rocky Mountain Scoliosis and Spine Center), Frank Schwab MD, PhD, Christopher I. Shaffrey $M D$ FACS (University of Virginia), Christopher P. Ames MD (University of California San Francisco Neuro Surgery), Gregory Mundis MD (San Diego Center for Spinal Disorders), Virginie Lafage PhD (NYU Langone Medical Center), Vedat Deviren MD (Univeristy of California San Francisco), Justin S. Smith MD PhD (University of Virginia Health System), Eric Klineberg MD (University of California - Davis), Behrooz A. Akbarnia MD, Douglas C. Burton MD, Robert Hart MD (Ohsu Spine Center), International Spine Study Group

Introduction: Drivers of patient satisfaction during fusion surgery are incompletely understood. To date, no large prospective evaluations of HRQoL measures and impacts of radiographic parameters on patient satisfaction following adult spinal deformity surgery have been reported. This multicenter prospective cohort study assessed correlations of HRQoL measures and radiographic parameters with patient satisfaction.

Methods: 157 adult patients undergoing fusion for adult spinal deformity was analyzed at minimum 2year follow-up. Correlation analyses were performed between Scoliosis Research Society Patient Questonaire-22 (SRS-22) satisfaction scores, and final and change from baseline, for Visual Analogue Scale (VAS) back/leg scores, (Oswestry Disability Index) ODI, SRS-22, and Short Form36 Mental Component Score (MCS) and Physical Component Score (PCS (SF36 MCS and PCS) scores. Satisfaction scores were also correlated to radiographic changes in sagittal vertical axis (SVA), coronal C7 plumbline, lumbar lordosis, pelvic tilt (PT). We also compared three patient groups: Highly Satisfied (HS; N=123, SRS$22>4.0$ ) and Less Satisfied (LS; N=34, SRS-22 < 4.0) and Not Satisfied (NS; N=9, SRS<2.5)- a subset of LS.

Results: Overall SRS-22 satisfaction scores were high (Mean 4.29 , range 1-5). There was a moderate correlation between satisfaction and final SRS-22 score $(\mathrm{r} 2=0.40)$, and a weak correlation with change in SRS-22 score, from baseline $(\mathrm{r} 2=0.32)$. All other HRQol data and radiographic parameters showed weaker correlations to SRS satisfaction scores ( $\mathrm{r} 2$ range $0.00001-0.27$ ). There were significant differences between HS and NS patients in all HRQols $(\mathrm{p}<.0001-.0089)$. There were no differences in final and change in radiographic parameters between HS, LS and NS patients.

Conclusions: Radiographic parameters have weak correlations to patient satisfaction. HRQoL scores are also weakly correlated, but differed between high, low and no satisfaction patients. Factors driving patient satisfaction appear to differ from surgical goals and current HRQoL measures. Other factors such as peri-operative expectations may be stronger drivers of patient satisfaction

108. Arthroplasty for Cervical Spondylotic Myelopathy: Similar Results to Patients Who Had Only Radiculopathy at ThreeYear Follow-up

Li-Yu Fay MD (Taipei Veterans General Hospital), Wen-Cheng Huang, Jau-Ching Wu MD (Department of Neurosurgery, Taipei Veterans General Hospital), Tzu-Yun Tsai MD, Chin-Chu Ko MD 


\section{Meeting Abstracts: AANS/CNS Section on Disorders of the Spine and Peripheral Nerves}

(Taipei Veterans General Hospital), Tsung-Hsi Tu MD (Veterans General Hospital Taipei), Ching-Lan Wu, Henrich Cheng (No. 322, Sec. 2, Shih-Pai Road, Peitou)

Introduction: Cervical arthroplasty has been accepted as a viable option for surgical management of cervical spondylosis or degenerative disc disease (DDD) with radiculopathy. However, it remains uncertain that patients who have cervical myelopathy caused by DDD can be equally managed with arthroplasty. This study aimed to evaluate the differences between patients with cervical spondylotic myelopathy and radiculopathy when treated by arthroplasty.

Methods: A total of 151 consecutive patients with cervical spondylotic myelopathy (CSM) or radiculopathy caused by DDD and who underwent one- or two-level cervical arthroplasty were enrolled. Clinical outcome measurements included the Visual Analogue Scale of neck and arm pain, Japanese Orthopedic Association scores, the Neck Disability Index, and the Nurick scores. Radiographic studies included lateral dynamic radiographs and computed tomography.

Results: The 125 patients $(82.8 \%)$ completed the follow-up for at least 24 months. There were 72 patients in the myelopathy group and 53 patients in the radiculopathy group. The mean age of the myelopathy group was approximately six years older than that of the radiculopathy group (53.1 vs. 47.2 years, $\mathrm{p}<0.001)$. The mean operation time, estimated blood loss, and perioperative analgesics were similar in both groups. There were significant improvements in clinical outcomes in the both groups. More than $92.5 \%$ of patients in this series retained spinal motion and there were no differences between the two groups $(\mathrm{p}=0.417)$. No secondary surgery was reported at the 3-year follow-up for both groups.

Conclusions: The severity of myelopathy improves after cervical arthroplasty in patients with CSM caused by DDD. At 3-year follow-up, the clinical and radiographic outcomes of cervical arthroplasty in DDD patients with CSM are similar to those patients who have only cervical radiculopathy. Therefore, cervical arthroplasty is a viable option for patients of CSM caused by DDD who require anterior surgery.

\section{Incidence and Prognostic Factors of C5 Palsy: A Clinical Study of 1,001 Cases and Review of the Literature}

Mohamad Bydon MD (Johns Hopkins Hospital), Mohamed Macki BA (Johns Hopkins University School of Medicine), Rafael De la Garza-Ramos MD (Johns Hopkins Hospital), Paul E. Kaloostian MD (The Johns Hopkins Hospital), Daniel M. Sciubba BS MD (Johns Hopkins University), Jean-Paul Wolinsky MD (Johns Hopkins University), Ziya L. Gokaslan MD (Johns Hopkins University), Ali Bydon MD (Johns Hopkins Hospital), Timothy F. Witham MD BS (Johns Hopkins Hospital)

Introduction: Postoperative C5 palsy is a motor decline in deltoid muscle function after spine surgery. The condition is historically associated with ossification of the posterior longitudinal ligament (OPLL) and laminoplasties. We present one of the first C5 palsy clinical studies excluding these conditions. Our objective is to determine the incidence and perioperative characteristics/predictors of C5 palsy after anterior versus posterior spinal decompression and fusions.

Methods: All patients undergoing C4-C5 operations for degenerative conditions were reviewed over 21 years. Anterior operations included an anterior cervical discectomy and fusion (ACDF) or a corpectomy, while posterior operations included laminectomy and fusion (with and without foraminotomies).

Results: Of the total 1,001 operations, $49.0 \%$ anterior and $51.0 \%$ posterior cases developed an overall C5 palsy incidence of 5.2\% (52 cases): $1.6 \%$ and $8.6 \%$, respectively $(\mathrm{p}<0.001)$. Of the 99 corpectomies, the palsy incidence of $4.0 \%$ was not only higher than ACDFs $(1.0 \%)(\mathrm{p}=0.058)$ but also followed an upward trend with increasing corpectomy levels $(\mathrm{p}=0.061)$. Of the 69 posterior and 83 anterior
C4-C5 foraminotomies ( $\mathrm{p}=0.130$ ), the incidence of $\mathrm{C} 5$ palsy was statistically higher in the posterior (14.5\%) versus anterior $(2.4 \%)$ cohort $(\mathrm{p}=0.006)$. Multivariate logistical regression identified age as the strongest predictor of $\mathrm{C} 5$ palsy in the anterior $(\mathrm{p}=0.020)$ and C4-C5 foraminotomy in the posterior $(\mathrm{p}=0.074)$ cohort. This condition improved within 3-6 months in $75 \%$ of patients in the anterior and $88.6 \%$ in the posterior cohort after a mean follow-up of 14.4 and 27.6 months, respectively.

Conclusions: In this large cohort examining postoperative C5 palsy, we found the posterior cohort had a statically higher incidence of C5 palsy. In anterior operations, cervical corpectomy had a higher incidence of $\mathrm{C} 5$ palsy relative to ACDF; however, age was the strongest predictor of $\mathrm{C} 5$ palsy. In posterior operations, C4-C5 foraminotomy carries the strongest risk.

\section{The NIS Database Does Not Accurately Reflect Surgical Indications for Fusion.}

Yakov Gologorsky MD (Mount Sinai Medical Center), John Chi MD, MPH (Brigham and Women's Hospital), Michael W. Groff MD FACS (Brigham and Women's Hospital)

Introduction: The rates of lumbar spinal fusion operations have increased dramatically over the past two decades, and several studies based on administrative databases such as the National Inpatient Sample (NIS) have suggested that the greatest rise is in the general categories of degenerative disc disease and disc herniation, neither of which is a well-accepted indication for lumbar fusion. The administrative databases classify cases with the International Classification of Disease, Version 9, Clinical Modification (ICD-9CM). ICD-9 discharge codes that are not generated by surgeons, but are rather assigned by trained hospital medical coders. It is unclear how accurately they capture the surgeon's indication for fusion. We sought to compare the ICD-9 code(s) assigned by the medical coder to the surgeon's indication based on a review of the medical chart (surgeon diagnosis).

Methods: A retrospective review was undertaken of all lumbar fusions performed at our institution by the department of neurosurgery between $7 / 1 / 2011$ to $8 / 31 / 2013$. Based on our review the indication for fusion for each case was categorized as: spondylolisthesis, deformity, tumor, infection, non-pathologic fracture, pseudarthrosis, adjacent level degeneration, stenosis, or degenerative disc pathology. These surgeon diagnoses were compared with the primary ICD-9 code that was submitted to the administrative databases.

Results: There were 179 lumbar fusion operations performed over 171 hospital admissions. We excluded 43 hospitalizations whose indications for fusion were tumor, infection, or non-pathological fracture believing that these indications for fusion are well accepted. There remained 128 admissions for lumbar fusion with degenerative diagnoses. The primary ICD-9 diagnosis matched the surgeon diagnosis in only 58/128 cases.

Conclusions: Characterizing indications for fusion based on ICD-9 codes does not accurately reflect the surgeon's indication. While administrative databases that use ICD-9 codes may accurately describe rates of lumbar fusion surgery, their role in identifying the indication for surgery is not well founded.

\section{Prospective Study of Biologic Disc Repair with NuQu Percutaneous Allogeneic Chondrocytes with Two-year Follow- up}

Domagoj Coric MD (Carolina Neurosurgery \& Spine), Kenneth Pettine MD, Margaret Boltes

Introduction: The purpose of the study was to prospectively evaluate the safety and initial efficacy of NuQu allogeneic juvenile chondrocyte treatment for biologic disc repair at 2-year follow-up. 


\section{Meeting Abstracts: AANS/CNS Section on Disorders of the Spine and Peripheral Nerves}

Methods: Patients were evaluated pre- and post-treatment at 1 , 3, 6, 12 and 24 months. Routine neurological examinations, MRI, SF-36, Oswestry Disability Index (ODI) and the Numerical Rating Scale (NRS) were performed. The quantitative change between baseline and 24 months were determined (significance levels were computed based on the paired t-test).

Results: Fifteen patients were treated with a single administration of $\mathrm{NuQu}$ juvenile chondrocytes. Levels: L3-4=2, L4-5=1, L5-S1=12. Pfirrman grade $\mathrm{III}=12$; grade $\mathrm{IV}=3$. Each treatment consisted of $1-2 \mathrm{cc}$ (mean volume $=1.3 \mathrm{cc}$ ) of juvenile chondrocytes $(\sim 10$ million cells $/ \mathrm{ml})$ with fibrin glue carrier. Mean peak pressure $=87.6$ psi. Twelve patients $(80 \%)$ were available for 2-year follow-up. The mean ODI (baseline $=53 ; 24$ months $=14.3 ; \mathrm{p}<0.0001$ ), NRS (baseline $=5.7 ; 24$ months $=2.5 ; \mathrm{p}=0.0036$ ) and SF-36 Physical scores (baseline $=35.3 ; 24$ months $=49.8 ; \mathrm{p}=0.0014$ ) all improved significantly from baseline. MRI: 9 (75\%) of 12 patients showed improvements in MRI imaging at 2 year follow. Four patients showed improvements in disc protrusions or height. Seven patients showed improvements in posterior high intensity zones (HIZ). No patients experienced neurological deterioration. There were no treatment related infections. There were no serious and unexpected adverse events. There was no observed immunological response to the chondrocyte treatment. Three patients $(20 \%)$ ultimately underwent total disc replacement due to persistent, but not worse than baseline, LBP.

Conclusions: The clinical and radiographic results from a prospective, cell-based, biologic disc repair study utilizing juvenile chondrocytes in the percutaneous treatment of lumbar spondylosis with mechanical LBP remain encouraging at two-year follow-up. Limitations of this study include small sample size and lack of control group. Further investigation with a prospective, randomized, double-blinded, placebo-controlled study is ongoing.

112. Efficacy and Safety of Surgical Treatment Cervical Spondylotic Myelopathy (CSM). Outcomes of the AOSpine Multicenter Prospective CSM-I Study

Michael G. Fehlings MD PhD FRCSC FACS (Toronto Western Hospital), Branko Kopjar, Helton Delfino MD (University of Sao Paulo- Ribeirao Preto), Shashank S. Kale, Giuseppe Barbagallo MD (Policlinico University Hospital), Ronald HMA Bartels MD PhD (Radboud University Medical Center), Qiang Zhou MD (Southwestern Hospital), Paul M. Arnold MD (Department of Neurosurgery), Mehmet Zileli MD (1421 sok 61-5 Gonca apt), Gamaliel Tan MD (Tan Tock Seng Hospital), Yasutsugu Yukawa MD, Osmar Moraes MD, Massimo Scerrati MD (Via Conca 71), Masato Tanaka MD, Tomoaki Toyone MD, Ciaran Bolger MD (Frenchay), Manuel Alvarado MD (Hospital San Juan de Dios)

Introduction: While studies from North America have suggested efficacy of surgery for cervical spondylotic myelopathy (CSM), little information is available regarding the international variations in clinical presentation, surgical approaches and outcomes of operative management for CSM.

Methods: A total of 479 patients with CSM were enrolled in a prospective multicenter, international study in 16 sites in Europe (5), Asia (6), North (2) and South America (3). One year post-surgery follow-up data on 447 (93.3\%) patients were analyzed for changes in the modified Japanese Orthopaedic Assessment scale (mJOA), Nurick Score, Neck Disability Index (NDI), Short Form 36v2, and complications.

Results: There were $35 \%$ females; average age 56.4 years (SD $11.91)$. Patients underwent anterior $(57.7 \%)$, posterior $(40.0 \%)$ or circumferential $(2.3 \%)$ surgery. There was a significant $(\mathrm{P}<0.001)$ improvement from baseline values to 12 months in all outcome measures. The mJOA improved from $12.48 \pm 2.84$ at baseline to $14.9 \pm 2.6$ at 12 months. The NDI improved from $37.33 \pm 19.91$ at baseline to $25.24 \pm 19.21$ at 12 months. The Nurick improved from $3.28 \pm 1.23$ to $2.00 \pm 1.58$. The SF36v2 PCS improved from $34.23 \pm 8.94$ to $41.18 \pm 10.55$ and the SF36v2 MCS improved from 39.52 \pm 13.03 at baseline to $46.22 \pm 13.25$ at 12 months. The 30 -meter timed walk test improved from $34.44 \pm 16.37$ pre-operatively to $29.35 \pm 13.44$ at 12 months. $77.0 \%$ patients experienced improvement in mJOA, $10.5 \%$ had no change and, $12.5 \%$ had decline. There were 7 cases of C5 radiculopathy, 14 dural tears, 24 cases of dysphagia 3 cases of dysphonia and 18 cases of progression of myelopathy.

Conclusions: This large prospective global clinical study shows that surgical treatment for CSM is associated with significant improvements in generic and patient-specific outcome measures at one year.

113. Comparison of Radiographic Results after Minimally Invasive (MIS), Hybrid (HYB) and Open (OPEN) Surgery for Adult Spinal Deformity (ASD): A Multicenter Study of 184 Patients

Richard G. Fessler MD (Rush University Medical Center), Raqeeb M. Haque MD (Cleveland Clinic Foundation), Yousef Ahmed, Michael Y. Wang MD FACS (University of Miami), Juan S. Uribe MD (University of South Florida - Division of Neurosurgery), David O. Okonkwo MD PhD (University of Pittsburgh), Neel Anand MD, Adam S. Kanter MD (University of Pittsburgh Medical Center Presbyterian), Frank La Marca MD (University of Michigan), virginie La Fage PhD (Orthopedic Surgery), Jamie Terran BS

Introduction: Treatment strategies for adult spinal deformity (ASD) include OPEN surgical techniques, and more recently, MIS applications with retroperitoneal lateral interbody fusion (LLIF) and MIS TLIF. We compared these techniques specifically targeting how radiographic parameters were affected.

Methods: Retrospective review of 2 prospective databases (MIS $\mathrm{n}=75$; OPEN $\mathrm{n}=109$ ) that were stratified into 3 groups: 1) cMIS $(\mathrm{n}=42)$ patients with LLIF or MIS TLIF and MIS pedicle screws; 2) HYB (n=33) - LIF with open posterior surgery and; 3) OPEN $(n=109)$. Inclusion criteria: coronal Cobb $>20^{\circ}$, age $>45 \mathrm{yrs}$, and minimum $1 \mathrm{yr}$ follow up. Paired, Unpaired t-tests and ANOVA was used to compare groups.

Results: MIS (63.9) and HYB (63.4y) were older than OPEN (60.4y, $\mathrm{p}=0.0001)$. Patients were $84.2 \%$ female. MIS had smaller preop $\left(32.1^{\circ}\right)$ and postop lumbar curves $\left(13.1^{\circ}\right)$ than OPEN $\left(43.2^{\circ}\right.$ to $\left.20.4^{\circ}\right)$ and $\operatorname{HYB}\left(44.3^{\circ}\right.$ to $\left.17.7^{\circ}\right)(\mathrm{p}<0.05)$. HYB had more lumbar curve correction $\left(26.6^{\circ}\right)$ than MIS $\left(18.8^{\circ}, \mathrm{p}=0.045\right)$. Preop lumbar lordosis (LL) was less in MIS (33.8) and HYB (31.9) than OPEN (42.7, $\mathrm{p}=0.025, \mathrm{p}=0.010)$. Postop LL was less in MIS (39.4) than either HYB (48.5) or OPEN (53.2, $\mathrm{p}=0.0001)$. Preoperative PI-LL was smaller for OPEN (12.3) than MIS (21.6) and HYB (22.03, $\mathrm{p}=0.018,0.026)$. Postop PI-LL for OPEN $(2.00)$ and HYB $(2.13)$ were smaller than MIS $(\mathrm{p}=0.0001,0.001)$. The change in PI-LL was larger for HYB (20.6) than OPEN (10.2) and MIS (5.5, $\mathrm{p}=0.023$, $0.003)$. SVA was higher in HYB and OPEN $(65,47 \mathrm{~mm})$ than MIS $(29 \mathrm{~mm} ; \mathrm{p}<0.05)$. The amount of SVA correction was greater for HYB and OPEN $(36,34 \mathrm{~mm})$ than MIS $(1 \mathrm{~mm})$. Pre and postop ODI and VAS were similar among the groups.

Conclusions: OPEN, HYB, and MIS techniques resulted in significant correction of coronal plane deformity. OPEN and HYB showed superior correction toward physiologic sagittal parameters compared to MIS techniques. MIS was unable to restore PT or PI-LL revealing limitations associated with MIS surgery. However, all groups achieved significant improvement in ODI and VAS.

114. Measurement and Optimization of Spinal Cord Perfusion Pressure in Spinal Cord Injury

Melissa C Werndle MBChB MRCS (St George's University of London), Samira Saadoun, Isaac Phang, Marek Czosnyka (Academic Neurosurgery Unit), Georgios V. Varsos, Zofia Czosnyka MD, 


\section{Meeting Abstracts: AANS/CNS Section on Disorders of the Spine and Peripheral Nerves}

Piotr Smielewski, Ali Jamous , B. Anthony Bell MD (Academic Neurosurgery Unit), Argyro Zoumprouli , Marios Papadopoulos C (St. George's University of London, Academic Neurosurgery Unit)

Introduction: To develop a technique for continuously monitoring intraspinal pressure at the injury site (ISP) after traumatic spinal cord injury (TSCI), analagous to intracranial pressure (ICP) after traumatic brain injury.

Methods: A pressure probe was placed subdurally at the injury site in 18 patients who had isolated, severe TSCI (ASIA A-C). ISP monitoring started within 72 hours of the injury and continued for up to a week. In four patients additional probes were inserted to simultaneously monitor subdural pressure below the injury and extradural pressure. Blood pressure was recorded from a radial artery catheter kept at the same horizontal level as the injured segment of the spinal cord. We determined the effect of various maneuvers on spinal cord perfusion pressure (SCPP) and spinal cord function, assessed using a limb motor score, motor evoked potentials (MEPs), ICG fluorescence and SPRX (an index of autoregulation).

Results: We recruited 34 subjects (18 with TSCI, 16 without). There were no procedure-related complications. ISP at the injury site was higher than subdural pressure below the injury or extradural pressure. Average ISP from the 18 TSCI patients was significantly higher than average ISP from 12 subjects without TSCI. Change in arterial pCO2, change in sevoflurane dose and mannitol administration had no significant effect on ISP or SCPP. Increase in inotrope dose significantly increased spinal cord perfusion pressure (SCPP). Bony realignment and laminectomy did not effectively lower ISP. Laminectomy was potentially detrimental by exposing the swollen spinal cord to compression forces applied to the skin. By intervening to increase SCPP, we could increase the amplitude of MEPs recorded from below or just above the injury level. In 2/2 patients with cervical ASIA C TSCI, higher SCPP correlated with increased limb motor score.

Conclusions: Our findings provide proof-of principle that it is feasible to measure subdural ISP at the injury site after TSCI.

\section{Circulating Tumor DNA as a Biomarker for Malignant} Peripheral Nerve Sheath Tumors

Chetan Bettegowda MD (Johns Hopkins Hospital), Allan J. Belzberg BSc MD FRCS(C) (Johns Hopkins School Of Medicine)

Introduction: Peripheral nerve sheath tumors (MPNSTs) are a major source of morbidity and mortality, especially in individuals with neurofibromatosis 1 , with five year survival rates reported to be as low as $20 \%$. There currently exists no good biomarker that can be used to monitor disease burden. Standard radiographic Methods: of measuring disease are often plagued by high cost, and poor sensitivity and specificity. We and others have previously demonstrated that many tumor types shed cell free DNA into the circulation. In this study, we determined whether MPNSTs shed detectable levels of tumor DNA (ctDNA) into the plasma. ctDNA is defined by the presence of tumor specific mutations not found in normal tissue.

Methods: We obtained tumor tissue and matched plasma from individuals with MPNST. The tumor was first sequenced for mutations and after at least one alteration was identified, the corresponding plasma was the queried for levels of that specific mutation. The abundance of the mutation in the plasma was a measure of ctDNA. ctDNA levels were quantified using a next generation sequencing approach coined Safe-SeqS.

Results: A bona fide somatic mutation was identified in all tumors tested. Using Safe-SeqS, the levels of ctDNA in the plasma ranged from undetectable to $>4 \%$.

Conclusions: MPNSTs shed detectable levels of ctDNA into the circulation and may form the basis for future personalized bio- markers. However, prospective studies are required to determine whether ctDNA can be used as a surrogate measure of disease burden. 116. Survival and Recurrence Following Resection of
Chordoma in the Mobile Spine: Results of 171 Patients from
the AOSpine Primary Tumor Knowledge Forum

Patricia L. Zadnik BA (The Johns Hopkins University School of Medicine), Peter Varga, Chetan Bettegowda MD (Johns Hopkins Hospital), Charles Fisher MD, Michael G. Fehlings MD PhD FRCSC FACS (Toronto Western Hospital), Nasir A Quraishi, Alessandro Luzzati, Laurence D. Rhines MD (University of Texas-MD Anderson Cancer Center), Jean-Paul Wolinsky MD (Johns Hopkins University), Daniel M. Sciubba BS MD (Johns Hopkins University), Mark B. Dekutoski MD (The Mayo Clinic), Norio Kawahara, Stefano Boriani MD (Rizzoli Institute,), Ziya L. Gokaslan MD (Johns Hopkins University)

Introduction: Chordomas are primary spinal tumors that grow via an indolent course but have devastating effects on patients' lives. The location of the tumor along the neuroaxis plays an important role in the feasibility of surgical resection. Boriani and colleagues published a series of 52 patients with mobile spine chordoma in 2006 reaffirming the importance of en bloc resection for this population. The present study reviews recurrence and survival data from 171 patients with mobile spine chordoma across multiple international centers.

Methods: A study coordinator was employed to assist with data collection, data entry, and capture of cross-sectional survival data. Descriptive statistics were summarized using mean/ standard deviation or frequency/ percentage. Mortality and time to tumor recurrence data was displayed by means of Kaplan-Meier curves. Cox regression was utilized for multivariate analysis.

Results: Fifty-seven patients (33\%) were female, 114 patients were male $(67 \%)$. The mean age at diagnosis was $58 \pm 14$ years (range 17-86 years). Median survival after surgery was 6.9 years for all patients. Surgeon's postoperative assessment of an en bloc resection $(\mathrm{p}=0.041)$ and Enneking appropriate resection $(\mathrm{p}=0.034)$ were associated with significantly increased survival. Patients with intralesional transgression on the surgical pathology specimen were 7.4 times more likely to experience a recurrence within 10 years (HR 7.442, $\mathrm{p}=0.002$ ). Age at diagnosis, tumor volume and level (cervical, thoracic, lumbar) did not significantly impact survival or tumor recurrence.

Conclusions: Achievement of en bloc resection with no evidence of contamination on the surgical pathology specimen affords increased survival and decreased risk of recurrence for patients with mobile spine chordoma. Patient age, tumor volume and the level of the lesion do not appear to influence patient survival or tumor recurrence.

117. Investigating Patient Perceptions: Analyzing the Role of Positive Spine MRI Findings on Patients' Perceived Need for Surgery

J. Nicole Bentley MD (University of Michigan), Patricia Yee, Kate Chang, Lynda Jun-San Yang MD PhD (University of Michigan Hospitals)

Introduction: Patient satisfaction and effective communication continue to punctuate the national healthcare debate, and these topics are increasingly being used as measures of clinician performance. It is imperative that neurosurgeons understand patients' perceptions and prior knowledge about their disorders before counseling them. Therefore, we surveyed a cohort of patients, presenting for routine evaluation to a neurosurgery spine clinic, to determine patients' baseline knowledge, expectations, and prior opinions regarding their spinal disorder. 


\section{Meeting Abstracts: AANS/CNS Section on Disorders of the Spine and Peripheral Nerves}

Methods: We administered a short questionnaire to patients referred to a general neurosurgery spine clinic with the chief complaint of 'back or neck pain,' prior to physician interaction. Questions assessed the role of imaging findings in shaping patients' perceptions of the need for surgery, and how they perceived surgical intervention as compared to conservative management in the context of back versus leg pain. Standard statistical Methods: were used for analysis.

Results: 121 consecutive participants were included. 52\% of patients reported they would be willing to undergo surgery solely for MRI abnormalities, even in the absence of attributable symptoms. $41 \%$ stated they would be willing to undergo surgery for asymptomatic X-ray abnormalities. 33\% thought that surgery was more effective than therapy for treating back pain. $21 \%$ of responders believed that steroid injections have more risk than surgery. Patient demographics, such as gender and education level, had no significant effect.

Conclusions: Patient misconceptions hinder surgeons' ability to manage expectations. Although the data presented here are preliminary, a surprisingly high percentage of patients demonstrate misconceptions regarding surgical treatment. Reasons behind these misconceptions are likely complex and warrant further investigation, but may be due to prior physician interactions or personally performed research. Understanding and acknowledging patient misconceptions remains important for improved physician communication/counseling for better outcomes after surgery.

\subsection{Haid Award: The Role of Sacral Slope in Lumbosacral Fusion - A Biomechanical Study}

Doniel Drazin MD MA, Mir Hussain BS, Matthew Phillips , John Hao , Terrence T. Kim MD, J. Patrick Johnson, MD MD (Spine Center), Brandon Bucklen PhD (Globus Medical Inc.)

Introduction: Sacral slope has correlated to progression of spondylolisthesis, yet there are limited biomechanical studies reporting its role in correction of adult spinal deformity, its influence on lumbosacral shear, and its importance when selecting instrumentation. This in vitro study investigates three anterior lumbar interbody fusion (ALIF) constructs in a biomechanics laboratory.

Methods: On a MTS Bionix (MTS,Eden Prairie,MN), ten healthy, fresh-frozen intact human lumbosacral vertebral segments were tested by applying an axial load of $550 \mathrm{~N}$ with sacral slope at $20^{\circ}$; testing was repeated at $10^{\circ}$ increments to $50^{\circ}$. Next, nine specimens (three segments per technique) were instrumented using one ALIF strategy: stand-alone implant with self-contained screws, pedicle screws (PSF) with a interbody spacer, plate placed from the anterior in a randomized order. Each instrumented specimen was tested at sacral slope $20^{\circ}$ with repeated testing to $50^{\circ}$ at $10^{\circ}$ increments. Linearly increasing axial loads, resulting in progressively higher shear and compressive stresses on the specimen. Load-deformation curves were collected. Parameters were recorded: deformation, ultimate load at failure, stiffness.

Results: Linear stiffness (N/mm) of L5-S1 motion segment measured at various angles of sacral slope: for ALIF+plate was $239.5 \pm 137.8\left(20^{\circ}\right), 251.9 \pm 164.7\left(30^{\circ}\right), 193.7 \pm 126.2\left(40^{\circ}\right)$, $148.5 \pm 114.7\left(50^{\circ}\right)$, for stand-alone construct was $208.4 \pm 115.9$ $\left(20^{\circ}\right), 202.3 \pm 114.2\left(30^{\circ}\right), 193.6 \pm 141.9\left(40^{\circ}\right), 150.3 \pm 102.9\left(50^{\circ}\right)$, and for ALIF+PSF was $217.7 \pm 139.7\left(20^{\circ}\right), 194.9130 .5\left(30^{\circ}\right)$, $133.2 \pm 86.8\left(40^{\circ}\right), 136.5 \pm 111.0\left(50^{\circ}\right)$. Trends in ultimate load and displacement were similar to linear stiffness. Axial compression across the disc space generally decreased with increasing sacral slope angle, indicating that sacral slopes past the $30^{\circ}$ threshold shifted L5-S1 motion into pure shear, instead of compressionshear.

Conclusions: At larger sacral slopes, the stand-alone ALIF appeared to be most stable in terms of stiffness and may play a larger role in preventing subsidence. Future work will investigate the clinical relevance of these biomechanical results.
201. Anterior vs. Posterior Surgical Approaches in Treatment of Cervical Spondylotic Myelopathy (CSM). Outcomes of the International AOSpine Multicenter Prospective CSM-I Study.

Michael G. Fehlings MD PhD FRCSC FACS (Toronto Western Hospital), Branko Kopjar MD, Helton Delfino MD (University of Sao Paulo- Ribeirao Preto), Shashank Sharad Kale MBBS MS MCh MD (All India Inst of Med Sciences), Giuseppe Barbagallo MD (Policlinico University Hospital), Ronald HMA Bartels MD PhD (Radboud University Medical Center), Qiang Zhou MD (Southwestern Hospital), Paul M. Arnold MD (Department of Neurosurgery), Mehmet Zileli MD (1421 sok 61-5 Gonca apt), Gamaliel Tan MD (Tan Tock Seng Hospital), Yasutsugu Yukawa MD, Osmar Moraes MD, Massimo Scerrati MD (Via Conca 71), Masato Tanaka MD, Tomoaki Toyone MD PhD (Teikyo University Chiba Medical Center), Ciaran Bolger MD (Frenchay), Manuel Alvarado MD (Hospital San Juan de Dios)

Introduction: While a number of excellent surgical options for cervical spondylotic myelopathy (CSM) exist, the comparative effectiveness of anterior vs posterior approaches remains hotly debated. Accordingly, we analyzed the data from a large, multicenter international study which examined surgical treatments for CSM to address this question.

Methods: A total of 479 patients with CSM were enrolled in a prospective multicenter, international study in 16 sites in North and South America, Europe and Asia with 467 receiving either Anterior $(\mathrm{N}=275)$ or Posterior $(\mathrm{N}=192)$ surgical approaches. One year postsurgery follow-up data on 435 (93.2\% follow-up) patients were analyzed for differences in outcomes between the anterior and posterior groups using the modified Japanese Orthopaedic Assessment scale (mJOA), Nurick Score, Neck Disability Index (NDI) and Short Form 36v2 outcome measures.

Results: Anterior patients were younger than posterior $(53.28 \pm 11.67$ and $60.78 \pm 10.96$, respectively, $\mathrm{P}<.01)$ and had more focal pathology (anterior: $2.89 \pm 2.78$ levels treated; posterior: $4.75 \pm 4.62$ levels treated $\mathrm{p}<.01)$. Both anterior and posterior patients improved on all outcome variables at 1 year post-operatively. There were no differences between the Anterior and Posterior groups in the extent of improvement in mJOA, NDI, SF36v2 Physical Component Score, SF36v2 Mental Component Score and times 30-meter walk test. The improvement in Nurick was greater in Anterior compared to Posterior patients $(1.38 \pm 1.41$ and $1.10 \pm 1.44$, respectively, $\mathrm{P}=0.045$ ).

Conclusions: There were important demographic and clinical differences between the anterior and posterior groups, suggesting that surgeons tailor the operative approach for CSM by virtue of the extent and location of pathology when these clinical and demographic factors were accounted for, anterior and posterior approaches were associated with similar improvements on all key outcome measures.

202. Intraoperative Assessment of Spinal Vascular Flow in the Surgery of Spinal Intramedullary Tumors Using Indocyanine Green Videoangiography

Toshihiro Takami MD (Osaka City University Graduate School of Medicine), Toru Yamagata, Kentaro Naito, Kenji Ohata MD (Osaka City University)

Introduction: To demonstrate the utility of indocyanine green videoangiography (ICG-VA) for intraoperative vascular flow assessment in the surgery of a variety of spinal intramedullary tumors to achieve an additional level of safety as well as precision with the surgical procedure.

Methods: Fourteen patients with spinal intramedullary tumors (nine cervical and five thoracic) operated on between August 2011 and April 2013 were included in the present study. A fluorescence 


\section{Meeting Abstracts: AANS/CNS Section on Disorders of the Spine and Peripheral Nerves}

surgical microscope was used to perform ICG-VA after standard exposure of the lesion to assess the dynamic flow of the spinal microvasculature.

Results: Twenty-seven ICG-VA injections were performed in 14 cases. Pathological diagnosis of the tumors included ependymoa, astrocytoma, cavernous malformation or hemagioblastoma. There were no complications or side-effects related to ICG-VA. Intraoperative ICG-VA provided dynamic flow images of the spinal microvasculature in accordance with the progress of surgical procedures. Angiographic images could be divided into arterial, capillary and venous phases. All angiographic images were well integrated into the microscopic view. The utility of ICG-VA could be summarized into three categories: 1) localization of normal spinal arteries and veins, 2) assessment of posterior spinal venous circulation, and 3 ) differentiation of feeding arteries, tumor and draining veins.

Conclusions: Intraoperative vascular flow assessment using ICG-VA was easy, repeatable, and practical without any significant procedure-related risks. ICG-VA can be used for careful analysis of spinal microvascular flow or anatomical orientation, which is necessary to ensure safe and precise resection of spinal intramedullary tumors.

\section{Responsiveness of Depression and Its Influence on Surgical Outcomes of Lumbar Degenerative Diseases}

Asdrubal Falavigna PhD (Leonilda Fassoli Zatti 201/01), Orlando Righesso, Alisson Teles, Pedro Guaresi Silva, Lucas Conzatti, Julia Bossardi, Joseph S. Cheng MD MS (Vanderbilt University Medical Center)

Introduction: Symptoms of depression are common in patients submitted to spine surgery due to degenerative disease. The impact of depression on the postoperative outcome has not been fully understood. To demonstrate the responsiveness of depression after surgery for lumbar degenerative disease and to verify the impact of this condition on surgical outcomes.

Methods: 91 patients with lumbar degenerative diseases were evaluated preoperatively, at 30 days and 1 year postoperatively with self-reported disability, depression, satisfaction index, fears and beliefs and health-related quality of life (HRQoL). Depression was assessed with the Beck Depression Inventory. According to depression responsiveness, the patients were classified into 4 groups: $\mathrm{NN}=$ no depression; $\mathrm{ND}=$ normal during the preoperative period and depression within 1 year; $\mathrm{DN}=$ depression during the preoperative period and normal within 1 year; $\mathrm{DD}=$ depression during the preoperative period and within 1 year.

Results: The prevalence of preoperative depression was $28.6 \%$. After surgery, $19.8 \%$ had depression within 30 days and $17.6 \%$ within 1 year postoperatively. Most patients with depression in the preoperative period recovered within 1-year postoperatively (65.4\%). Poor preoperative HRQoL measures were associated with higher rates of responsiveness of depression during the followup. Patients who presented depression at the 1 year postoperative evaluation had a worse functional outcome than the patient who did not have depression. The patient who improved from depression had a similar outcome to those who did not present depression throughout the study. Dissatisfaction within 1 year postoperatively was greater in patients who become depressed after surgery and remained depressed at 1 year follow-up than in those who were already depressed in the preoperative period and did not improve after surgery $(\mathrm{NN}=8.8 \% ; \mathrm{ND}=42.9 \% ; \mathrm{DN}=17.6 \% ; \mathrm{DD}=44.4 \%$; $\mathrm{P}=0.012$ ).

Conclusions: Most patients with depression in the preoperative period recover within 1 year postoperatively. Responsiveness of depression is clearly associated with surgical outcomes. The presence of depression after the surgical treatment independent of when it begins had a major negative impact on prognosis which reinforces the need to screen for depressive symptoms not only prior to but after spine surgery.
204. Regenerative Electrodes Enable Functional Electrical Stimulation of Peripheral Motor Axons and Distal Musculature

Wilson Zachary Ray MD (Washington University School of Medicine), Erik Zellmer, Daniel Moran PhD, Matthew R MacEwan BSE PhD (Washington University School of Medicine)

Introduction: Development of advanced neuroprosthetic systems depend on the creation of a microelectrode device capable of selectively interfacing peripheral nerve tissue. One device capable of achieving such an interface is the regenerative sieve electrode. Unfortunately, sieve electrodes are dependent upon robust, successful nerve regeneration through the device. [1] Therefore, we hypothesized that the incorporation of fibrin-based delivery systems loaded with GDNF into sieve electrode assemblies will enhance nerve regeneration through the electrode and provide superior nerve/ electrode interface capabilities in vivo. [2]

Methods: Custom-designed sieve electrodes were fabricated out of polyimide and gold using sacrificial photolithography. (Fig. 1) Prior to implantation in the sciatic nerve of male Lewis rats, silicone conduits attached to the electrodes were filled with either fibrinbased delivery system loaded with $100 \mathrm{ng} / \mathrm{ml}$ GDNF, or saline. Post-operatively, functional nerve regeneration through implanted devices and neural interfacing capabilities were assessed in situ via nerve conduction studies and evoked muscle force measurement upon sciatic nerve stimulation. Regenerated nerve segments were terminally explanted and fixed, for histomorphometric evaluation.

Results: Histological analysis demonstrated that sieve electrode assemblies containing GDNF-loaded matrices supported greater numbers of nerve fibers and improved functional recovery, CNAP conduction, and muscle force production compared to saline controls. Sieve electrode assemblies containing GDNF further supported stable interfaces with multiple groups of regenerated motor axons over $>5$ months, allowing for graded recruitment of multiple muscles within the lower leg. Implanted sieve electrodes assemblies containing GDNF demonstrated a threshold of motor activation of $\sim 10 \mathrm{uA}$, were capable of recruiting $99.8 \%$ of regenerated motor units, and demonstrated $99.5 \%$ selectivity of activation for all independent muscle tested.

Conclusions: The present study demonstrates that controlled delivery of trophic factors modulates neural activity at the tissue/ electrode interface, supporting future investigation into devices capable of simultaneously interacting with peripheral nerve tissue through bioelectric and biomolecular stimuli.

\section{Anterior Approaches for Symptomatic Central Herniated Thoracic Dises}

Justin Charles Clark BS MD (Barrow Neurological Institute), Mark E. Oppenlander MD (Barrow Neurological Institute), Curtis A. Dickman MD (Neurosurgical Associates)

Introduction: Anterior approaches are required to safely remove symptomatic central herniated thoracic discs (HTDs) when they are compressing the spinal cord. This is the largest report in the literature comparing thoracoscopy to open thoracotomy for the treatment of central HTDs.

Methods: We retrospectively reviewed a prospectively-maintained surgical database that contained the senior author's experience with surgically treating central HTDs.

Results: Between 1994 and 2012, 183 patients underwent 184 anterior approaches for central HTDs causing myelopathy, $(\mathrm{N}=74)$, myelopathy and radiculopathy $(\mathrm{N}=53)$ or thoracic radiculopathy $(\mathrm{N}=54)$. Access to the ventral thoracic spine was achieved using an open thoracotomy $(\mathrm{N}=48)$ or thoracoscopic approach $(\mathrm{n}=136)$. Patients treated via open thoracotomy had more extensive pathology than patients chosen for thoracoscopy; a greater average of levels treated (1.8 vs 1.2), more patients with calcified and/or ossified discs 


\section{Meeting Abstracts: AANS/CNS Section on Disorders of the Spine and Peripheral Nerves}

(72.9\% vs $19.7 \%)$, as well as giant $(62.5 \%$ vs $4.4 \%)$ or transdural disks $(10.4 \%$ vs $1.5 \%)$. The open thoracotomy patients were also more likely to require internal fixation with spinal instrumentation (93.8\% vs $13.9 \%)$. Thoracotomy patients had significantly greater surgical blood loss $(1,466 \mathrm{cc}$ vs $330 \mathrm{cc})$, longer hospital stays $(8.8$ days vs 4.8 days) and more pulmonary complications requiring intervention ( $27.1 \%$ vs $8.0 \%$ ). Follow-up for thoracotomy (mean = 48.4 months) and thoracoscopy (mean $=35.5$ months) demonstrated that thoracotomy patients were more likely to have improved at least 1 Frankel grade during the post-operative period (79.2\% vs $47.4 \%)$.

Conclusions: Thoracoscopy and open thoracotomy each provide unique surgical solutions for patients presenting with symptomatic central HTD. Thorough understanding of the benefits of each approach will ensure optimal patient outcomes.

206. Kineflex|C Cervical Arthroplasty: Long-term Clinical and Radiographic Outcomes from a Prospective, Randomized IDE Study

Domagoj Coric MD (Carolina Neurosurgery \& Spine), Richard D. Guyer MD, Pierce D. Nunley MD, Margaret Boltes

Introduction: The purpose of this paper is to present the longterm results of the prospective, randomized Kineflex/C (K-C) cervical total disc replacement (cTDR) IDE study comparing cTDR to anterior cervical discectomy and fusion (ACDF) for the treatment of cervical radiculopathy at one level, C3-7.

Methods: A total of 347 patients were enrolled at 21 sites: 193 patients in the investigational $\mathrm{K}-\mathrm{C}$ treatment group (136 randomized and 57 non-randomized) and 133 subjects in the ACDF control group (all randomized). Patients were evaluated pre-operatively and post-operatively at $1.5,3,6,12$ months and at yearly intervals out to 60 months. Evaluations at each visit included routine neurological examinations, $x$-rays, the Neck Disability Index (NDI), arm and neck pain assessments (VAS) and the SF-36.For all clinical outcome measurements the quantitative change between baseline and 60 months were determined and the significance levels were computed based on the paired t-test.

Results: One-hundred and seventy-six patients were available for 5 year follow-up for an overall follow-up rate of 65\% (93 K-C and 83 ACDF patients). The overall success rate was significantly greater in the K-C group (77\%) vs. ACDF group (58\%). There were more supplemental surgeries at the index level in the ACDF group $(\mathrm{n}=11,8 \%)$ compared to the K-C group $(\mathrm{n}=10,7 \%)$. There were low subsidence $(n=3,1.4 \%)$ and migration $(n=2,0.9 \%)$ rates in the K-C cohort. Only two K-C patients ( $0.9 \%$ ) exhibited bridging heterotopic ossification without motion at 5-year follow-up. ROM was maintained in the K-C patients (pre-op= 8.2 degrees, 60 months $=10.6$ degrees) but not in the ACDF patients (pre-op $=7.6$ degrees, 60 months $=0.7$ degrees). There was a statistically greater degree of radiographic degeneration at adjacent levels in the ACDF patients compared to the arthroplasty patients $(\mathrm{p}=0.001)$.

Conclusions: Five year clinical and radiographic data from the Kineflex-C prospective, randomized IDE study confirm safety and efficacy at long-term follow-up. The Kineflex-C cTDR achieved statistically significant improvements in clinical outcomes while maintaining motion and showing less adjacent level radiographic deterioration.

207. Two Level Corpectomy versus Three Level Discectomy of the Cervical Spine: Analysis of Spinal Alignment, Adjacent Level Disease, Neck Pain, and Neurologic Function

Darryl Lau (University of California, San Francisco), Dean Chou M.D. (University of California San Francisco), Praveen V. Mummaneni MD (University of California San Francisco Spine Center)

Introduction: In the treatment of degenerative disease of the cervical spine, both corpectomy and/or discectomy are commonly performed. However, it remains to be determined whether multilevel corpectomy or multilevel discectomy is able to offer the best outcomes. In this study, we compared 2-level corpectomy against 3-level ACDF by assessing spinal alignment, adjacent level disease, pain, and neurologic function.

Methods: Between 2006 and 2011, all patients who had 2-level corpectomy or 3-level ACDF for degenerative disease were identified. EBL and complications were recorded. Outcomes of spinal alignment, adjacent segment disease, (VAS) neck pain, and Nurick scores were examined. Spinal alignment was measure utilizing Cobb angles. Adjacent segment disease was defined as any adjacent level disease requiring steroid injections and/or surgery. Fischer's exact test and student's two tailed t-test were used to compare the two groups.

Results: 29 patients underwent a 2-level corpectomy and 35 patients underwent a 3-level ACDF. In the corpectomy group, mean age was 56.5 years and $66.6 \%$ were male. In the discectomy group mean age was 59.3 years and $40.0 \%$ were male. There were no significant differences in preoperative comorbidities, pain, Nurick score, and spinal alignment between the two groups. Mean followup was 20.0 months and 21.7 months for the 2 level corpectomy group and the 3 level ACDF groups, respectively. 2 level corpectomy was associated with significantly higher blood loss compared to 3 level ACDF ( $326.2 \mathrm{cc}$ vs. $130.0 \mathrm{cc}, \mathrm{p}=0.004)$. Overall complication rate for 2 level corpectomy was $10.3 \%$ and overall complication rate for 3 level ACDF was 8.6\%; this difference was not statistically significant $(\mathrm{p}=0.999)$. Also, there were no significant differences in follow-up VAS neck pain score (1.2 vs.1.1, $\mathrm{p}=0.945)$, Nurick score (1.1 vs. $0.6, p=0.444)$, correction of spinal alignment (11.1 degrees vs. 8.3 degrees, $\mathrm{p}=0.475)$, and rate of adjacent level disease $(6.9 \%$ vs. $5.7 \%, \mathrm{p}=0.869)$.

Conclusions: These findings suggest 2-level corpectomy and 3-level ACDF offer similar outcomes in regards to spinal alignment, adjacent segment disease, neck pain, and neurologic function. However, the group that underwent corpectomy had greater blood loss.

208. Stand Alone Lateral Interbody Fusion (saMIS) vs Circumferential Minimally Invasive Surgery (cMIS) for Adult Spinal Deformity (ASD)

Adam S. Kanter MD (University of Pittsburgh Medical Center Presbyterian), David O. Okonkwo MD PhD (University of Pittsburgh), Bryan D Bolinger DO (University of Pittsburgh Medical Center), Gregory Mundis MD (San Diego Center for Spinal Disorders), Juan S. Uribe MD (University of South Florida Division of Neurosurgery), Michael Y. Wang MD FACS (University of Miami), Praveen V. Mummaneni MD (University of California San Francisco Spine Center), Neel Anand MD, Richard D. Fessler MD (Keystone Medical Center - Novi), Paul Park MD (University Of Michigan, Department of Neurosurgery), Kai-Ming G. Fu MD PhD (Weill Cornell Medical College), Virginie Lafage PhD (NYU Langone Medical Center), Jamie Terran BS, Behrooz A. Akbarnia $M D$, Vedat Deviren MD (Univeristy of California San Francisco), International Spine Study Group

Introduction: Minimally invasive surgery for ASD is gaining popularity with the premise of reducing surgical complication rates. Lateral lumbar interbody fusion (LLIF) is one such advance, often supplemented by posterior MIS pedicle screws (PPI). Controversy exists whether MIS LIF requires PPI to enhance correction, promote stiffness for arthrodesis, and improve outcomes.

Methods: A multicenter retrospective analysis of prospectively collected data of 45 patients, from 7 sites. Inclusion criteria: age $>45$ years with minimum of $20^{\circ} \mathrm{Cobb}$ angle and 1 year follow-up. In cMIS, patients underwent LLIF followed by PPI; in saMIS, patients underwent transpsoas LLIF without PPI. 


\section{Meeting Abstracts: AANS/CNS Section on Disorders of the Spine and Peripheral Nerves}

Results: Mean follow-up was 29 mos for cMIS (N=39) and saMIS ( $\mathrm{N}=6)$. Preoperatively, there were no differences between cMIS and saMIS in age (63.6 vs 63.6yrs), gender, or radiographic parameters (lumbar $\mathrm{Cobb}=32^{\circ}$ vs $34.5^{\circ} ; \mathrm{SVA}=2.9 \mathrm{~cm}$ vs $3.1 \mathrm{~cm}$; PI-LL $=21^{\circ}$, respectively for cMIS and saMIS). saMIS patients had a higher ASA grade (2.8 vs 1.9, $\mathrm{p}=0.015)$, more co-morbidities ( 3.7 vs $1.5, \mathrm{p}=0.015)$. More saMIS patients were revision cases $(66.7 \%$ vs. $15.4 \%, \mathrm{p}=0.016)$. The cMIS group exhibited significant Cobb angle correction $\left(31^{\circ}\right.$ to $\left.10^{\circ}, \mathrm{p}<0.001\right)$ and increase in lumbar lordosis $\left(34^{\circ}\right.$ vs $\left.40^{\circ}, \mathrm{p}<0.001\right)$. saMIS did not. Both groups had significant improvements in ODI (41 pre-op to 23 post-op for CMIS; and 43 to 29 for saMIS), with no difference between groups.

Conclusions: Patients treated with saMIS had greater medical problems and history of previous spine surgery. In this study, addition of PPI to MIS (LLIF) results in better correction compared to saMIS procedures alone. Despite greater disability and previous surgery rates, the saMIS group demonstrated equivalence in clinical outcomes, but limited deformity correction. Long-term follow-up is essential to evaluate patients that did not meet the intended radiographic goals as long-term success has been associated with global sagittal and coronal balance.

\section{Pain, Disability and the Surgeon's Recommendation Against Surgical Intervention May Negatively Impact Satisfaction Scores in Patients with Spinal Disorders}

Marcus D. Mazur MD (University of Utah), Sara M. McEvoy (University of Utah), Meic H. Schmidt MD FACS (Clinical Neuosciences \& Spine Center), Erica F Bisson

Introduction: Patient satisfaction is becoming a widely used metric to measure health care quality. Patients often bring expectations to medical encounters and satisfaction correlates with the extent to which physicians fulfill patient expectations. Because satisfaction scores are right-skewed, even small differences in mean scores can carry significant weight. Little information, however, is available on the specific factors that impact satisfaction in patients with spinal disorders.

Methods: We conducted a retrospective study of adult patients who underwent a new spine consultation to determine the effect of pain and disability self-assessments and the surgeon's recommendation on patient satisfaction scores. Patients completed the Oswestry Disability Index (ODI) prior to their clinic appointment and a Press Ganey Patient Satisfaction Survey after their visit. Satisfaction scores were graded in 25-point increments from 0 (very poor) to 100 (very good). Wilcoxon rank sum test was used to evaluate the association between patient satisfaction and current pain and disability self-assessment. We also investigated whether the surgeon's recommendation against surgery would negatively impact patient satisfaction.

Results: Of the 164 patients, who completed satisfaction surveys, 58 had completed the ODI and 124 had received a recommendation for or against surgery. Mean satisfaction scores were lower among patients who had self-assessed severe and moderate disability than those with mild disability $(78.9,84.0$, and 91.6 , respectively; $\mathrm{p}=0.026$ ). Patients who received a recommendation against surgery reported lower mean satisfaction scores with respect to their surgeon than those who received a recommendation for surgery (92.4 vs. 97.2; $\mathrm{p}=0.010$ ).

Conclusions: High pain and disability self-assessment and the surgeon's recommendation against surgical intervention are associated with lower satisfaction scores in patients with spinal disorders.

210. Regeneration of Rabbit Intervertebral Discs Using A Single Low-Concentration Injection of Human Disc Progenitor Cells

Kevin T. Foley MD FACS (Semmes-Murphey Neurologic \& Spine Institute), Lara Ionescu Silverman PhD (DiscGenics, Inc.), Galina
Dulatova (DiscGenics), Terry Tandeski PhD (Discgenics, Inc), Subba Chintalacharuvu PhD, Tricia Wolff (Covance, Inc), Jairo Nunes DVM PhD ACVP, Antwain Howard DVM DCLAM, Kellie Howard (Covance, Inc), Mark Parrish (Covance, Inc)

Introduction: One approach to reversing degenerative disc disease (DDD) is to supply the disc with a cell population that results in the production of additional extracellular matrix. Our group is developing injectable cell therapy with human disc-derived progenitor cells and a viscous scaffold carrier. Laboratory study has shown these cells to be immunologically privileged (e.g. no HLA-DR/DQ surface antigens). This study assessed the efficacy and safety of a range of single doses of human discogenic cells in 24 rabbits followed for 10-14 weeks.

Methods: Using a validated model of DDD, annular puncture was performed in 24 New Zealand white rabbits (3 discs/rabbit). Two weeks later, $3,000,10,000,30,000$ or 100,000 human discogenic cells in $25 \mu 1$ of hyaluronic acid were injected into injured discs. Injured control discs were maintained and scaffold carrier alone was injected into other discs ( $\mathrm{n}=12$ discs/condition). Disc height index (DHI) was measured at baseline and then periodically using $\mathrm{x}$-ray over the course of 10 weeks (12 animals) and 14 weeks (12 animals). Histology and gene expression analysis were performed at necropsy. Body weights, clinical observations and serology were used to monitor safety.

Results: 14-week data is presented. No safety concerns were noted. DHI improved from $62 \%$ to $77 \%$ (STD 14\%) by 6 weeks with all cell doses but not with carrier alone. The lowest dose, 3,000 cells, improved DHI to $80 \%$ at 6 weeks and $85 \%$ by 14 weeks. DHI differences between injured controls and cell-treated discs were statistically significant via 2-way ANOVA and Tukey's post-hoc test. Histology revealed improvement of rabbit disc morphology with human cell treatment.

Conclusions: This study confirmed findings from pilot studies and showed safety and efficacy of a single intradiscal dose of human progenitor cells in a rabbit model of DDD. Interestingly, the greatest efficacy was noted at relatively lower cell doses.

211. C5 Palsy Following Posterior Cervical Decompression and Fusion: Cost and Quality of Life Implications

Jacob A Miller BS (Cleveland Clinic Lerner College of Medicine), Thomas Mroz, Daniel Lubelski, Matthew D. Alvin MA, Edward C. Benzel MD (Cleveland Clinic Foundation)

Introduction: C5 palsy is a debilitating postoperative complication of cervical decompression surgery. While the prognosis is typically good, patients may be unable to perform basic activities of daily living, decreasing their quality of life. No studies have investigated the quality of life or financial implications. In the present study, we measure the impact of C5 palsy upon patients' quality of life and determine the associated costs of recovery following C5 palsy.

Methods: A 2:1 matched retrospective cohort study was conducted of individuals that had undergone posterior cervical decompression and fusion (PCDF) at the Cleveland Clinic. Controls were matched on age, gender, BMI, and diagnosis. Demographic, operative, postoperative, quality of life, and cost data were collected for both the C5 palsy and control groups, with 1-year follow-up.

Results: We reviewed 245 patients that underwent PCDF, and identified 17 (6.9\%) with C5 palsy. No significant differences in demographic or operative characteristics were observed between the two groups. The C5 palsy group had a significantly reduced capacity for self-care in the immediate postoperative period ( $2.0 \pm 0.7$ vs. $1.2 \pm 0.4, \mathrm{p}<0.001)$ and long-term (1.6 \pm 0.6 vs. $1.2 \pm 0.4, \mathrm{p}=0.004)$, and a significantly reduced capacity for completion of usual activities ( $2.4 \pm 0.7$ vs. $1.9 \pm 0.6, \mathrm{p}=0.014)$. Furthermore, the $\mathrm{C} 5$ group had a significantly greater cost of physical/occupational therapy, an increase 


\section{Meeting Abstracts: AANS/CNS Section on Disorders of the Spine and Peripheral Nerves}

of $\$ 2078$ ( $\$ 4386 \pm 2801$ vs. $\$ 2307 \pm 1907, p=0.013)$. There were no significant differences in any other postoperative cost. Overall, there was a significantly greater cost $(\$ 1918)$ in total postoperative care for the C5 palsy group $(\$ 7584 \pm 3992$ vs. $\$ 5666 \pm 2359$, $\mathrm{p}=0.038$ ).

Conclusions: This study represents the first quantification of the quality of life and financial impact of C5 palsy. We found that C5 palsy adds a significant burden upon patients' quality of life, particularly in self-care and completion of usual activities. Furthermore, C5 palsy presents a financial burden to the healthcare system in cost of rehabilitation.

\section{Spinal Cord Fiber Optic Monitoring}

Robert M. Galler DO (Department of Neurosurgery), Thomas F Floyd MD (Stony Brook University Hospital), Thomas Bilfinger MD SCD (Stony Brook University Hospital), Rickson Mesquita PhD (University of Pennsylvania), Angela D'Souza PhD (Stony Brook University), Arjun Yodh PhD (University of Pennsylvania)

Introduction: Monitoring for the detection of spinal cord ischemia is considered an important adjunct for both spine \& vascular surgery, as spinal cord ischemia may be amenable to treatment if detected early. Current Methods: employed to detect spinal cord ischemia, based upon electrophysiology, are indirect, temporally insensitive, nonspecific, and cumbersome. We have developed a linearly arrayed, prototypical fiber optic device based on Diffuse Correlation Spectroscopy (DCS) and Diffuse Optical Spectroscopy (DOS) principles that allow for the detection of changes in spinal cord blood flow and oxygenation

Methods: In adult Dorsett sheep, femoral and carotid arterial lines were placed. An intra-aortic balloon was advanced to the common cephalic root. A left atrial catheter was placed for the injection of microspheres for spinal cord blood flow measurement. A fogarty arterial embolectomy balloon catheter was inflated directly onto the spinal cord to create crush injury. The fiber optic probe was placed via percutaneous (16-G Touhy needle) \& open (laminectomy) approaches. Spinal cord blood flow and oxygenation responses to pharmacologic interventions (phenylephrine and nitroprusside) and aortic occlusion were measured at multiple levels.

Results: Data from this and previous experiments demonstrates that the fiber optic probe is sensitive, successfully detecting changes in spinal cord blood flow associated with acute hypertension in $12 / 12$ trials $(100 \%)$, acute hypotension in $9 / 9$ trials (100\%), aortic clamping in 14/14 trials (100\%), and epidural balloon inflation onto the spinal cord in $2 / 2$ trials. In a series of experiments, the mean change in flow associated with the hypertensive and hypotensive challenges was (+) $51 \pm 11 \%$ and (-) $39 \pm 11 \%$ respectively. The instantaneous flow:pressure response correlation was $\mathrm{R} 2=0.92, \mathrm{p}<.001$. Rapid pressure autoregulation was seen with pressure changes $>50$ torr.

Conclusions: Figure 4 - Preclinical studies in our lab indicate the probe is sensitive, accurate, and immediate - Probe can be placed similar to epidural catheters - Probe materials have been tested to be biocompatible There is an opportunity for pre and post-operative continuous unattended monitoring There may be improvement in surgical planning and overall safety

213. Adjacent Level Ligamentous Injury Associated with Traumatic Cervical Spine Fractures: Indications for Magnetic Resonance Imaging and Implications for Treatment

Basem I. Awad MD (Mansoura University Hospital), Margaret Carmody MD (Case Western Reserve/Univ. Hospitals of Cleveland), Daniel Lubelski, Jeffry MD Claridge , John J. Como MD (MetroHealth), Thomas E. Mroz MD (Cleveland Clinic -
Neuroscience Institute), Timothy A. Moore MD (MetroHealth Medical Center), Michael P. Steinmetz, MD (MetroHealth Medical Center- H910)

Introduction: Magnetic Resonant Imaging (MRI) is a vital tool for detection of instability and soft tissue trauma following cervical spine injuries (CSIs). However, high cost, prolonged imaging time, and limited use in hemodynamically unstable patients necessitates that the utility of MRI on all CSI patients be scrutinized.

Methods: A retrospective review was performed of all patients treated for a CSI at a Level I trauma center between 2005 and 2010. Patients were identified using CT imaging and a subgroup underwent MRI. Adjacent level ligamentous injury (ALLI) was defined as suraspinous and interspinous ligaments and classified concerning the fracture level. We identified correlations between fracture type, level and treatment strategy to develop guidelines for necessary MRI use.

Results: MRI was performed on 240/787 patients. Evidence of soft tissue injury was identified in $54.6 \%$ of these patients. ALLI was the most common soft tissue injury occurring in 80/240 of cases, which were then subdivided to either above (31/80), below $(31 / 80)$ or both above and below $(18 / 80)$ the concurrent fracture level. Patients with ALLI were significantly more likely to have injured C3 $(\mathrm{p}<0.01)$ and $\mathrm{C} 5(\mathrm{p}<0.03)$, and present in association with widened disc space ( $13 \%$ versus $4 \%, \mathrm{p}=0.03)$ and multiple CSI (1.7 versus $1.5, p=0.008)$. None of the patients who presented with ALLI above and below, and underwent surgical fixation, had the whole ALLI levels included in the fixation strategy. Contrary, respectively $100 \%$ and $87 \%$ of patients with ALLI only above and only below, had the whole ALLI included.

Conclusions: MRI found an associated soft tissue injury in only $50 \%$ of imaged patients. Multiple fractured cervical levels, fractures particularly at $\mathrm{C} 3$ and $\mathrm{C} 5$, and widened disc space should all raise the treating physician's level of suspicion for ALLI. Our data shows MRI directed treatment has substantial value in a select group of patients.

\section{Sacroplasty for Tumor-associated Sacral Insufficiency} Fractures

Nelson Moussazadeh MD (New York Presbyterian Hospital (Cornell Campus)), Timothy Werner, Patrick Boland M.D., Ilya Laufer MD (Memorial Sloan-Kettering Cancer Center), Eric Lis, Mark H. Bilsky MD (Memorial Sloan Kettering Cancer Center)

Introduction: Tumor-associated sacral insufficiency fractures (SIF) present a significant clinical challenge. As survivability increases for many malignancies, sacral fractures associated with metastases, sacral or extended pelvic radiation and paraneoplastic osteoporosis are increasingly common and yet remain difficult to treat in the setting of the unacceptable morbidity of open sacral surgery. We describe our prospective experience with sacroplasty for tumor-associated SIF, including the largest series to date of radiation-induced SIF.

Methods: Twenty-five patients aged 32-84 with intractable sacral or referable radicular pain and imaging evidence of SIF were included. Six patients suffered from baseline urinary/fecal incontinence. Thirty-one percutaneous sacral cement augmentation procedures were performed with a median 5.8cc of PMMA or Cortoss (Stryker, Michigan USA) under fluoroscopic guidance and with concurrent biopsy acquisition. Seventeen patients had undergone previous pelvic or sacral radiation and were found to have no viable tumor; 4 patients had active lytic lesions; and 2 patients had other potential oncologic-related etiologies for osteoporosis (steroid dependence and oophorectomy). Patients were monitored for postoperative pain reduction, procedural including neurologic morbidity, and functional outcomes.

Results: Twenty of 25 patients (80\%) had reduction in their visual analog pain score (VAS) at a median follow-up of 6.5 months; no 


\section{Meeting Abstracts: AANS/CNS Section on Disorders of the Spine and Peripheral Nerves}

patients worsened. Mean VAS declined from 8.8 to 4.7 postprocedurally $(\mathrm{p}<0.001)$, with significant reductions regardless of underlying pathology (viable tumor, pelvic/local radiation without identified tumor, paraneoplastic or unidentified; $\mathrm{p}<0.05)$. At last follow up, 6 patients required fewer ambulatory aids and 3 patients were newly ambulatory. Eighteen procedures resulted in extravertebral cement migration; however, no instance was clinically relevant. Six repeat procedures were performed: 1 for persistent symptoms with subsequent improved pain control, 4 for recurrent pain with progressive fracture, and 1 for contralateral augmentation. No morbidity was encountered.

Conclusions: Sacroplasty is a safe and effective option for the palliation of sacral insufficiency fractures in the oncologic population.

215. Routine Use of Recombinant Human Bone Morphogenetic Protein-2 in Posterior Fusions of the Pediatric Spine and Incidence of Cancer

Christina Sayama MD MPH (Texas Children's Hosptial, Baylor College of Medicine), Matthew Willsey BS, Alison Brayton ADN (Baylor Pediatric Neurosurgery), valentina briceno RN, Sheila Ryan JD, Thomas G. Luerssen BS, MD, FACS, FAAP (Clinical Care Center), Andrew Jea MD (Texas Children's Hospital)

Introduction: The purpose of this study is to determine the safety of recombinant human bone morphogenetic protein-2 (rhBMP-2) use in posterior instrumented fusions of the pediatric population, focusing on cancer risk. Our previous study reported the short-term (mean follow-up of 11 months) safety and efficacy of rhBMP-2 in the pediatric age group. We now report our long-term results with a minimum of 24 months followup.

Methods: A retrospective review of 57 consecutive pediatric patients who underwent posterior occiptocervical, cervical, thoracic, lumbar, or lumbosacral spine fusion from October 1st, 2007 to June 30th, 2011 at Texas Children's Hospital was performed. 13 patients were excluded from further analysis because of loss of follow-up or follow-up was less than 24 months. 2 patients died during the follow-up period and were divided into a separate cohort.

Results: The average age was 10 years, 7 months (range, 0 years, 9 months to 20 years, 0 months). The mean follow-up was 46.3 months (range, 24-70 months). Cancer status was determined at most recent encounter with the patient and/or caretaker(s), in-person during a clinic visit or through telephone follow-up. 24 months after administration of rh-BMP-2, there were no cases of new malignancy, or degeneration or metastasis of existing tumors in our small cohort of pediatric patients. Moreover, the definitive cause of death was determined in 2 of our 2 patients that died during the study period; none of the deaths were related to BMP nor the development, degeneration, or metastasis of cancer.

Conclusions: Despite the large number of adult studies reporting positive effects of BMP on cancer, our long-term outcomes using rhBMP-2 in the pediatric population suggest that it is a safe adjunct to posterior spine fusions of the occipitocervical, cervical, thoracic, lumbar, and lumbosacral spine. There were no new cases of cancer, or degeneration or metastasis of existing malignancies in our series of children after 24 months of follow-up.

216. - Withdrawn. Optimal Duration of Conservative Management Prior to Surgery for Cervical and Lumbar Radiculopathy: A Literature Review

Vincent J Alentado BS, Daniel Lubelski, Michael P. Steinmetz MD (MetroHealth Medical Center- H910), Edward C. Benzel MD (Cleveland Clinic Foundation), Thomas E. Mroz MD (Cleveland Clinic - Neuroscience Institute)

Introduction: The review the natural history, outcomes and cost- effectiveness studies relating to the current standard of waiting 6 weeks prior to performing surgery on patients with various spinal pathologies.

Methods: A systematic Medline search from 1953 to 2013 was performed to identify natural history, outcomes and costeffectiveness studies relating to the optimal period of conservative management prior to surgical intervention for both cervical and lumbar radiculopathy. Demographic information, method of obtaining results, operative indication and clinical outcomes are reviewed for each study.

Results: A total of five thousand seven hundred and nineteen studies were identified; of these 13 studies were selected for inclusion. Natural history studies demonstrated that $88 \%$ of patients with cervical radiculopathy and $70 \%$ of patients with lumbar radiculopathy had symptom improvement within 4 weeks following onset of symptoms. Outcomes and cost-effectiveness studies supported surgical intervention within 8 weeks of symptom onset for both cervical and lumbar radiculopathy.

Conclusions: There are limited studies supporting any optimal duration of conservative treatment prior to surgery for various spinal pathologies, and therefore evidence-based conclusions cannot be made. Based on the available literature, this review suggests that an optimal timing for surgery following cervical radiculopathy is within 8 weeks following onset of symptoms but that a shorter time period of 4 weeks may be appropriate based on natural history studies. Additionally, this review suggests that an optimal timing for surgery following lumbar radiculopathy is between 4 and 8 weeks. However, due to the heterogeneity of the studies reviewed, a prospective study is needed to explicitly identify the optimal duration of conservative therapy prior to surgery so that costs may be reduced and patient outcomes improved.

\section{The Predictive Value of Transcranial Magnetic Stimulation} of the Motor Cortex in Cervical Myelopathy

Marcus D. Mazur MD (University of Utah), Sara M. McEvoy (University of Utah), Angela White PhD, Erica F Bisson

Introduction: Selecting patients with cervical spondylotic myelopathy (CSM) who would benefit from surgery is complicated by the lack of objective assessment tools with high predictive value. Transcranial magnetic stimulation (TMS) is a painless and noninvasive modality used to objectively measure central motor conduction. We investigated the utility of TMS in predicting functional outcomes in patients undergoing surgery for CSM.

Methods: We conducted a prospective clinical trial of patients undergoing surgical intervention for CSM. Baseline TMS results and functional assessments were obtained preoperatively and at 3, 6 and 12 months postoperatively. TMS results were measured as central motor conduction time (CMCT) to the upper extremity. Functional assessments included Nurick grade, mJOA score, 10 meter walk, grasp and release test and 9-hole peg test. Spearman rank-order correlation was used to evaluate the association between baseline TMS results and baseline functional assessment to establish normative TMS values in patients with CSM. Repeated measures analysis of variance was performed with an autoregressive correlation structure to determine the effect of baseline TMS on postoperative functional assessments to evaluate the predictive value of TMS.

Results: 17 patients with CSM were included (mean age 60 years, range 42 to 78), of whom 10 underwent anterior surgery, 4 had posterior surgery, and 3 had combined anterior/posterior surgery. Mean CMCT decreased from $9.7 \pm 4.9$ at baseline to $8.5 \pm 3.5$ at 6 months. Severity of myelopathy based on mJOA score inversely correlated with CMCT (Pearson's r=-0.614, $\mathrm{p}=0.011$ ). Preoperative CMCT correlated with postoperative performance on the 10 meter walk $(\mathrm{r}=0.31, \mathrm{p}=0.014)$, grasp and release test $(-0.82, \mathrm{p}=0.010)$, and the 9-hole peg test $(0.58, \mathrm{p}=0.022)$.

Conclusions: Transcranial magnetic stimulation may provide 


\section{Meeting Abstracts: AANS/CNS Section on Disorders of the Spine and Peripheral Nerves}

useful parameters for determining disease severity and predicting functional outcomes in patients with cervical myelopathy.

\section{Superior Cluneal Nerve Entrapment Neuropathy after Posterior Lumbar Spinal Surgery}

Masahiko Akiyama MD DMSc (Fuji City General Hospital), Yuichi Sasaki MD (Jikei University School of Medicine), Hiroki Ohashi MD (Jikei University School of Medicine), Satoshi Tani MD

Introduction: Superior cluneal nerve ( $\mathrm{SCN}$ ) neuropathy has been reported as a causative factor in low-back pain, one of the complications of harvesting bone from the posterior iliac crest for grafting. However, recent evidence has suggested that SCN neuralgia was more commonly the result of a spontaneous entrapment rather than a nerve injury during bone harvest. Here we present 6 cases of SCN entrapment neuropathy after lumbar spinal surgery without posterior iliac bone grafting.

Methods: The criteria for a diagnosis of SCN entrapment neuropathy are newly developed low-back pain involving the iliac crest and buttock after posterior lumbar spinal surgery without bone grafting, a trigger point over the posterior iliac crest, and numbness and radiating pain in the $\mathrm{SCN}$ region when the trigger point is compressed.

Results: We diagnosed 6 cases of SCN neuropathy, 3 men and 3 women, their age from 45 to 80 . There were 4 cases of simple decompression, 1 case of MIS-TLIF and 1 case of MIS decompression and fusion for trauma. No cases were involved of posterior iliac bone graft. SCN occurred 6 days to 32 months after surgery; 2 cases within 10 days (early) and 4 cases 2 months after surgery (delayed). Three cases had unilateral and the other 3 cases had bilateral symptoms. In 2 early cases, neuralgia resolved with one nerve block procedure, however, multiple nerve block and longtime pain medication needed in 4 delayed cases.

Conclusions: SCN entrapment neuropathy has been underestimated among the spine surgeons and must be considered if patients experience newly developed low-back pain after lumbar spinal surgery.

\section{Intra-Operative Epidural Anesthetic Injection For Control Of Immediate Post-Operative Pain In PACU After Lumbar Spinal Surgery}

\section{Fred H. Geisler MD PhD (Chicago Back Institute)}

Introduction: Lumbar surgery patients often develop severe low back spasms immediately after emerging from anesthesia in the PACU and often require IV narcotics. This severe operative site pain can take hours to several days to resolve, often causes the patient significant discomfort, and can delay discharge - especially from planned outpatient surgery. This study is a retrospective chart review of posterior lumbar surgical patients in the PACU.

Methods: These post-operative muscle spasms and/or operative site pain in the PACU have been considered inevitable consequences of lumbar surgery for most patients. This study examines the addition of an intra-operative epidural anesthetic injection to decrease the maximum pain in the PACU and hence the number of I.V. doses of narcotics required. A consecutive series of 168 decompressive and/or in situ arthrodesis lumbar surgeries compared as to the use $(n=133)$ or not $(n=35)$ of a single epidural injection at skin closure via an intra-operatively placed catheter. Maximum pain response (0-10 VAS) and number of narcotic doses required were measured in the PACU. The effect of a single epidural injection of $2 \mathrm{cc}$ Fentanyl and $8 \mathrm{cc}$ of $1 / 4 \%$ Marcaine given near the end of wound closure via an epidural catheter inserted intraoperatively was investigated and compared with similar patients not receiving an epidural injection.

Results: The maximum pain score recorded in the PACU was $5.3 / 10$ without the epidural and $1.7 / 10$ with the epidural injection $(p<0.0001)$. The percentage of patients with no lumbar pain $(0 / 10$ pain score) at any time in the PACU was $20 \%$ for the group without and $65 \%$ for the group with the epidural injection $(\mathrm{p}<0.0001)$. The number of patients requiring IV or IM narcotics (83\% versus $22 \%$ ) and the number of doses (1.8 versus 0.3$)$ for the without and with epidural groups, respectively, were both statistically significant $(p<0.0001)$. There were no complications noted related to the use of the epidural injection.

Conclusions: A single intra-operative single epidural injection appears safe and effective in postoperative pain management after lumbar surgery in the PACU by significantly decreasing both the initial post-operative site pain and lumbar muscle spasms as the patients emerge from general anesthesia.

\section{Evaluation of CSF Dynamics via Cine Flow MRI Following Extradural Chiari I Decompression}

Jennifer L Quon BS (Yale University School of Medicine), Ryan A Grant MD (Yale University School of Medicine), Michael L. DiLuna MD (Yale Department of Neurosurgery)

Introduction: Extradural decompression is a technique for treating Chiari I malformation that avoids the complications of dural opening. Mounting evidence demonstrates that extradural decompression effectively treats clinical symptoms, with a minimal reoperation rate. Neurologic symptoms may be secondary to obstructed flow of cerebrospinal fluid (CSF), but successful extradural decompression results in improved CSF dynamics. We advocate that phase-contrast cine flow MRI be employed pre- and post-surgery to assess improvement in flow dynamics, and therefore a measure of satisfactory decompression.

Methods: We describe a surgical series of 11 patients with Chiari I malformation undergoing extradural decompression, for which improvement of CSF flow was evaluated with phase-contrast cine flow MRI. Ten of the eleven patients received standard pre- and post-operative MRIs, 4 patients also had pre- and post-operative phase-contrast cine, while the remainder had only post-operative cine.

Results: All 11 patients presented with symptomatic Chiari I malformation, confirmed on imaging to have tonsillar herniation without syringomelia. All patients underwent suboccipital decompression and C1-laminectomy with splitting of the outer dural leaflet. Eight out of eleven (72.7\%) patients showed partial or complete resolution of their neurologic symptoms at initial follow-up. Of these eight patients, six (75.0\%) demonstrated improved CSF flow on post-operative phase-contrast cine MRI, with one missing pre-operative MRI for comparison. All three of the patients without clinical improvement also lacked radiologic improvement. There was one post-operative complication of dysphagia and dysphonia. None of the patients have required a second operation.

Conclusions: Extradural decompression has the potential to be the first-line treatment for Chiari I malformation, but lacks an objective measure by which to assess surgical success. Cine flow MRI non-invasively demonstrates CSF dynamics and may be associated with resolution of clinical symptoms. Including cine imaging as part of routine evaluation can help identify which patients are most likely to benefit from surgery.

221. PPAR-d Complements the Benefits of Surgical Decompression in a Novel Mouse Model of Cervical Spondylotic Myelopathy

Spyridon K Karadimas MD PhD (Toronto Western Hospital), Michael G. Fehlings MD PhD FRCSC FACS (Toronto Western Hospital)

Introduction: While successful surgical intervention can arrest the progression of CSM, most patients are left with significant residual neurological impairment. Recent evidence shows that the 


\section{Meeting Abstracts: AANS/CNS Section on Disorders of the Spine and Peripheral Nerves}

chronic cervical spinal cord compression disrupts the blood spinal cord barrier (BSCB) and causes endothelial cell loss. PPAR-d is known to preserve endothelial cell and promotes BSCB integrity in ischemia. Here, we hypothesized that increased PPAR-d signaling would protect the spinal cord microvasculature and provide complementary benefits to surgical decompression in a CSM mouse model.

Methods: The experimental design is depicted in Figure 1. Decompression was achieved by removal of the C6 lamina and the compression formation underneath it. Gait analysis was performed using CatWalk. Immunohistochemistry, western blot and PCR were also performed. To evaluate the BSCB integrity Evans-blue injection was performed. ANOVAs were used for statistical analysis.

Results: All groups have MRI confirmation of similar degrees of cord compression. At 6 weeks post-decompression $\mathrm{D}+\mathrm{G}$ group showed significant increased hindlimb and forelimb stride length compared to $\mathrm{G}, \mathrm{D}$ and $\mathrm{C}$ groups ( $\mathrm{p}<0.05$ for each comparison). Similarly, the forelimb and hindlimb stance phase duration was significantly decreased in $\mathrm{D}+\mathrm{G}$ group compared to the $\mathrm{G}, \mathrm{D}$ and $C$ groups $(p<0.05$ for each comparison). These results indicate that the combinatorial treatment attenuated forelimbs and hindlimb spasticity compared to decompression alone treatment. Moreover, decreased BSCB disruption, increased neuronal and endothelial cell number were found to $\mathrm{D}+\mathrm{G}$ group compared to decompression treatment $(\mathrm{p}<0.05)$. Moreover, PPAR-d administration preserves the endothelial cells and the BSCB integrity under the chronic compression (Fig. 2). Finally the administration of PPAR-d agonist decreased the oxidative damage caused by decompression surgery $(\mathrm{p}<0.05$ for DR vs $\mathrm{D})$.

Conclusions: These results demonstrate that PPAR-d agonism has microvasculo- and neuro-protective effects. Finally in synergy with decompression leads to better functional outcomes than the decompression alone in CSM.

\section{An FDA IDE Clinical Trial through 48 Months: Cervical TDR versus ACDF}

Michael S. Hisey, Reginald J. Davis MD, Hyun Bae MD (The Spine Institute), Kee Kim MD, Steven E. Gaede MD, Pierce D. Nunley MD, Donna D. Ohnmeiss Dr.Med.

Introduction: One-level total disc replacement (TDR) has been shown as safe and effective as ACDF for treating symptomatic degenerative disc disease. Clinical data from a single level TDR trial is presented through 4-year follow up.

Methods: A prospective, randomized, concurrently controlled clinical trial was conducted at 23 centers. Randomization for surgery was in a 2:1 ratio with 164 patients being treated with the investigational device (Mobi-C ${ }^{\circledR}$ Cervical Disc Prosthesis) and 81 with ACDF with an anterior plate and allograft bone. Outcomes at 6 weeks, 3, 6, 12, 24, 36 and 48 months included NDI, VAS neck and arm pain, subsequent surgery rates, and adjacent segment degeneration.

Results: Follow-up at 48 months was $77 \%$ for the randomized patient population. Evenly matched at baseline, significant improvement in NDI and VAS neck and arm pain scores was observed at each follow-up period through 48 months. Mean improvement in NDI was $38 \pm 19$ for the TDR population and $34 \pm 17$ for ACDF. The TDR group improved on average $52 \pm 30$ for VAS neck pain compared to $50 \pm 30$ for the ACDF group. VAS arm pain scores; the TDR group averaged improvement of $56 \pm 36$ compared to ACDF with an average improvement of $57 \pm 31$, following the worst scoring arm at baseline. The subsequent surgery rate was significantly higher in the ACDF group (9.9\%) compared to the TDR group $(3.0 \%, \mathrm{p}=0.03)$. Additionally, the incidence of adjacent segment degeneration was significantly higher in the ACDF group at both the inferior and superior adjacent segment (inferior segment: TDR: $30 \%$ vs. ACDF: $50 \%, \mathrm{p}=0.013$; superior segment: TDR: $34 \%$ vs. ACDF: $53 \%, \mathrm{p}=0.011$ ).
Conclusions: Significant improvement was observed in both pain and function for both groups, while TDR demonstrated significantly lower subsequent surgery and adjacent segment degeneration rates compared to the ACDF group. TDR at longer follow-up continues to be a safe and effective alternative to ACDF.

223. Cost-utility Analysis of Anterior Cervical Discectomy and Fusion with Plating (ACDFP) versus Posterior Cervical Foraminotomy (PCF) for Patients with Single-level Cervical Radiculopathy at 1-year Follow-up

Matthew D. Alvin MBA MA (Case Western Reserve Unviersity School of Medicine), Daniel Lubelski , Kalil G. Abdullah (University of Pennsylvania), Robert G. Whitmore MD (Lahey Spine Program), Edward C. Benzel MD (Cleveland Clinic Foundation), Thomas E. Mroz MD (Cleveland Clinic - Neuroscience Institute)

Introduction: Cervical radiculopathy is commonly treated by either posterior cervical foraminotomy (PCF) or anterior cervical discectomy and fusion with plating (ACDFP). The present study analyzes the cost effectiveness of ACDFP versus PCF for patients with single-level cervical radiculopathy.

Methods: 45 patients who underwent ACDFP and 25 patients who underwent PCF were analyzed. 1-year postoperative health outcomes were assessed based on Visual Analogue Scale (VAS), Pain Disability Questionnaire (PDQ), Patient Health Questionnaire (PHQ-9), and EuroQol-5 Dimensions (EQ-5D) questionnaires. Direct medical costs were estimated using Medicare national payment amounts, and indirect costs were based on patient missed work days and patient income. Postoperative 1-year cost/utility ratios and the incremental cost effectiveness ratio (ICER) were calculated to assess for cost effectiveness using a threshold of $\$ 100,000 /$ QALY gained.

Results: The ACDFP cohort showed improvement in VAS, PDQ, and EQ-5D scores whereas the PCF cohort did so for the EQ-5D at 1 year postoperatively $(\mathrm{p}<0.01)$. No significant difference existed between ACDFP and PCF cohorts for any health outcomes. The 1-year cost-utility ratio for the PCF cohort was significantly lower (\$79,856/QALY gained) than that for the ACDFP cohort $(\$ 142,100 /$ QALY gained) $(\mathrm{p}<0.01)$. In calculating the 1-year ICER, ACDFP was found to be dominated by PCF.

Conclusions: Statistically and clinically significant improvements in quality of life measures were seen 1 year postoperatively for both cohorts. While ACDFP led to greater improvements in quality of life as compared to PCF for patients with single-level cervical radiculopathy, ACDFP is not cost effective relative to the threshold of $\$ 100,000 / \mathrm{QALY}$ gained at 1 year postoperatively and is dominated by PCF.

224. Outcomes of Lumbopelvic Fixation with S2-Alar-Iliac Screws or Iliac Bolts

Marcus D. Mazur MD (University of Utah), Vijay Ravindra MD (University of Utah Hospital), Meic H. Schmidt MD FACS (Clinical Neuosciences \& Spine Center), Darrel S. Brodke MD, Brandon Lawrence, Andrew T. Dailey

Introduction: S2-alar-iliac (S2AI) screws are an attractive alternative to conventional fixation with iliac bolts since they are lower profile, require less muscle dissection, and have greater pullout strength. Few studies, however, compare outcomes between these techniques.

Methods: We conducted a retrospective study of adult patients who underwent lumbopelvic fixation to determine the risk factors for failure after placement of S2AI screws or iliac bolts. Failure was defined as the need for revision arthrodesis or instrumentation, radiographic nonunion or hardware-related complications. Univariate, multivariate, and survival analyses were used to compare patients who had failure with those who did not. 


\section{Meeting Abstracts: AANS/CNS Section on Disorders of the Spine and Peripheral Nerves}

Results: Of the 53 patients included, 23 received S2AI screws. Failure-free survival rate was $92.5 \%$ at 1 year, $85.9 \%$ at 2 years, and $78.2 \%$ at 3 years. Failure was more common in patients with iliac bolts than those with S2AI screws (9 vs. 2; $\mathrm{p}=0.026$ ). Four patients with iliac bolts developed nonunion at L5-S1. Placement of iliac bolts (OR 12.8, 95\% CI [1.81, 191.42]), number of levels fused (1.6, $[1.15,2.40])$, and age $(0.9,[0.78,0.97])$ were significantly associated with failure. Reason for pelvic fixation, pelvic screw length, number of previous fusion operations, smoking, and use of BMP were not associated with surgical outcomes.

Conclusions: High rates of fusion at L5-S1 can be achieved in patients undergoing lumbopelvic fixation with either S2AI screws or iliac bolts. Early results suggest that patients with S2AI screws have a trend towards lower failure rates and fewer reoperations than those with iliac bolts.

\section{Allograft vs. Autograft in Occipitocervical Fusion: Radiological and Clinical Outcomes}

Jakub Godzik B.A. (Washington University School of Medicine), Vijay Ravindra MD (University of Utah Hospital), Wilson Zachary Ray MD (Washington University School of Medicine), Erica Fay Bisson MD FAANS (University of Utah Health Care), Meic H. Schmidt MD FACS (Clinical Neuosciences \& Spine Center), Andrew T. Dailey

Introduction: We compare fusion rates between structural allograft and autograft, evaluate correction of radiographic parameters, and describe symptom relief with each technique.

Methods: Adult patients treated with occipitocervical fusions between 2003 and 2010 were reviewed. Outcomes include radiological fusion, radiographic measurements of $\mathrm{C} 1-\mathrm{C} 2$ and $\mathrm{C} 2-\mathrm{C} 7$ lordosis angles; C2-C7 sagittal vertical alignment (SVA); posterior occipitocervical angle (POCA), and patient outcome parameters.

Results: 44 consecutive patients underwent single-stage rigid posterior OCF between 2003 and 2010. 33 patients underwent OCF with allograft, and 11 (25\%) underwent OCF with autograft. Eight patients treated with autograft had at least 12 months of follow-up, and all $(8 / 8)$ demonstrated solid bony fusion. Of 20 patients treated with allograft that had 12 months of follow-up, 18 (90\%) demonstrated solid bony fusion at final follow-up (autograft vs. allograft fusion, $\mathrm{p}=0.353$ ). Mean preoperative $\mathrm{C} 1-\mathrm{C} 2$ lordosis was $34^{\circ}$ (range $22-53^{\circ}$ ), with a mean C2-C7 lordosis angle of $18^{\circ}$ (range $-7-50^{\circ}$ ). The C2-C7 SVA ranged from 7 to $61 \mathrm{~mm}$ (mean $27 \mathrm{~mm}$ ), and the mean preoperative POCA was $100^{\circ}$ (range $\left.73-121^{\circ}\right)$. There was no statistically significant difference in preoperative measurements between the two cohorts. Across all patients, the C2-C7 SVA was correlated with $\mathrm{C} 1 / \mathrm{C} 2$ lordosis (Pearson' $\mathrm{s}=0.499, \mathrm{p}=0.008$ ) and POCA ( $r=-0.531, p=0.004)$. Mean neck pain VNRS improved significantly in both groups (5.9 to 3.2 for allograft, $\mathrm{p}<0.001 ; 7.3$ to 3.3 for autograft, $\mathrm{p}=0.005)$. Mean NDI improved in both groups $(57 \%$ to $30 \%$ for allograft, $n=8, p=0.055 ; 54.6 \%$ to $39 \%$ for autograft, $n=3$, $\mathrm{p}=0.28$. The rate of graft-site related complications in the autograft group was $9 \%(1 / 11)$.

Conclusions: The use of allograft in occipitocervical fusion allows for a high rate of successful arthrodesis and comes with similar radiographic and clinical outcomes versus autograft, yet avoids the potentially significant morbidity associated with autograft harvesting.

226. Remembering What the Doctor Said: Review of a Single Surgeon's Experience Video Recording Doctor-Patient Visits Using The Medical Memory

Randall W. Porter MD (Division of Neurosurgery), Andrew J. Meeusen MA LIS (Barrow Neurosurgical Associates)

Introduction: Patients considering surgery often have difficulty remembering all of the information presented to them in the con- sultation and follow-up appointments with their doctors. Critical information may be forgotten or misremembered in the days or weeks after a visit and can adversely affect both the patient's decision-making capabilities and their post-surgical care. The goal of this study was to review the experience of a single surgeon with personalized video recording to improve patient-doctor communication and help patients remember all of their doctor's instructions after leaving the office.

Methods: Personalized video recordings were offered to patients of a single neurosurgeon between November, 2009 and September, 2013. Patients were given the opportunity to have their visit with the physician recorded and then uploaded to a secure website, and given access to the video to watch and listen to again at home. The collected surveys of patient experiences with their videos and their ability to remember the information discussed in the visit after having watched the video.

Results: Between 2009 and 2013, 1,078 videos were recorded in a single neurosurgeon's office. 346 of these patients were sent an electronic survey to ask about their impression of the service. 143 responses were collected $(41.3 \%)$. Over $90 \%$ of patients watched the video at least once. $69 \%$ of patients shared their video with another person. $48 \%$ of respondents felt more at ease, $35 \%$ reported reduced anxiety, and $65 \%$ of patients also felt like they remembered more of what their doctor told them after viewing the video.

Conclusions: Patients who cannot or do not remember their doctor's instructions fully after leaving their visit are at risk of not following those instructions. This greatly impacts the patient's quality of care. Video recording doctor-patient conversations can help patients not only follow all instructions, but also improve their physical health.

\section{New Findings in Lipomatosis of Nerve: A Review of $\mathbf{5 2}$ Cases Evaluated at One Institution}

Mark Mahan MD (Barrow Neurological Institute), Robert J. Spinner MD (Mayo Clinic)

Introduction: Lipomatosis of nerve (LN), also known as fibrolipomatous hamartoma, is a condition of massive nerve enlargement due to fibrofatty proliferation. It is also variably associated with nerve-territory overgrowth. Although numerous case studies exist, there is little data on large populations, associated findings or longterm follow-up.

Methods: Review of the searchable records for LN at our institution found 52 cases, confirmed by pathology or pathognomonic appearance on MRI. Clinical histories were reviewed to identify patterns of disease extent or similarities of treatment.

Results: Several new clinical associations have been discovered: 1. Natural history: LN progressively enlarges, disproportionate to skeletal growth. Growth is most dramatic in youth. 2. Surgical intervention: Nerve injury or transection appears to produce fibroproliferative neuromas. These unusual and distinct neuromas may also enlarge with time. 3. Pathoanatomy: $\mathrm{LN}$ is not isolated to the limbs, but may occur in the axial skeleton and abdominal viscera, leading to unusual conditions of lipomatosis and hypertrophy. LN may be associated with benign tumors, such as lipomas and osteochondromas, suggesting an interconnected relationship.

Conclusions: LN is a unique disorder of nerves that has impressive consequences for the surrounding innervated soft tissues and bone. We have identified an expanded set of relationships that may shed insight into nerve-related growth.

\section{Wrong Level Surgery in the Thoracic Spine: A Survey Based Evaluation}

Eric A. Potts MD, Justin S. Smith MD PhD (University of Virginia Health System), Christopher I. Shaffrey MD FACS (University of 


\section{Meeting Abstracts: AANS/CNS Section on Disorders of the Spine and Peripheral Nerves}

Virginia), Praveen V. Mummaneni MD (University of California San Francisco Spine Center), Michael W. Groff MD FACS (Brigham and Women's Hospital), Joseph S. Cheng MD MS (Vanderbilt University Medical Center)

Introduction: Wrong level spine surgery continues despite national efforts by regulators and professional societies. Causes of wrong level surgery in the spine remain not well understood or characterized. We undertook this study to further understand the occurrence of wrong level surgery in the thoracic spine and characterize best practices to avoid this error.

Methods: A survey regarding localization and wrong level surgery in the thoracic spine was sent to all members of the Joint Section of the Disorders of the Spine and Peripheral Nerves. Twenty four questions addressed localizing a radio-lucent lesion in the thoracic spine (e.g. soft disc herniation). Respondents with wrong level surgery had eight further questions specifically about errors made and remediation taken.

Results: There were 473 responses from 905 requests $(52 \%)$. 109 surgeons (23\%) performed 167 wrong level cases. Most commonly surgeons had one or two episodes of wrong level surgery, $63(58.3 \%)$ and $34(31.4 \%)$ respectively. Data on 148 wrong level cases was available and combined. $80(54.1 \%)$ cases were done posteriorly; 28 (18.9\%) far lateral (e.g. costotransversectomy); and $39(26.4 \%)$ lateral. Surgeons were asked what errors were made. The most common error was poor imaging due to body habitus in 80 cases (38.6\%). Surgeon error in counting was present in $68(33.2 \%)$ cases. When comparing the surgeons who have performed wrong level surgery to those who have not, there was only one question with statistically significant difference, which was the rate of using lateral fluoroscopy versus anterior-posterior projections. The group with wrong level surgery relied on AP fluoroscopy more frequently $(\mathrm{p}=0.05)$.

Conclusions: Despite the scrutiny on wrong level surgery, it occurs at a relatively high rate. Further study of this potentially preventable error and development of standardized protocols to decrease its occurrence is warranted.

\section{MAYFIELD CLINICAL SCIENCE}

229. No Difference in Postoperative Complications, Pain and Functional Outcomes Up to 2 Years After Incidental Durotomy in Lumbar Spinal Fusion: A Prospective, Multi-Institutional, Propensity Matched Analysis of 1,741 Patients

Owoicho Adogwa M.D. M.P.H (Duke University Medical Center, Division of Neurosurgery), Mary I. Huang MD (Duke University Hospital), Paul Thompson BS (Division of Neurosurgery), Timothy Darlington, Parastou Fatemi, Terence Verla (Duke University School of Medicine), Joel Martin , Oren N. Gottfried MD (Duke University Mecical Center), Greg Anderson MD, Robert E. Isaacs $M D$ (Duke University Medical Center)

Introduction: Incidental durotomies occur in up to $17 \%$ of spinal operations. Controversy exists regarding the short and long-term consequences of durotomies. In this study, we assess long-term outcomes and complications after incidental durotomies for patients undergoing primary lumbar spinal fusions.

Methods: 1,741 patients were selected from a multi-institutional prospective data registry. All patients underwent primary lumbar spinal fusion for low back pain and/or radiculopathy between January 2003 and December 2010. We collected and analyzed data on patient demographics, postoperative complications, back pain (BP-Visual Analog Scale), leg pain (LP-Visual Analog Scale), and functional disability (Owestry Disability Index) over two years, with risk adjusted propensity score modeling. We compared two patient cohort: those with incidental duromoties and those without incidental durotomies.

Results: Incidental durotomies occurred in 70 patients (4\%).
Compared to the control group $(\mathrm{n}=1671)$, there was no significant difference in postoperative infection $(\mathrm{p}=0.32)$, need for reoperation $(\mathrm{p}=0.85)$, or symptomatic neurologic damage $(\mathrm{p}=0.66)$. At one and two year follow up, there was no difference in patient reported outcomes of back pain (BP-Visual Analog Scale), leg pain (LP-Visual Analog Scale), or functional disability (Owestry Disability Index) $(p>0.3)$, with results remaining consistent in the propensity-matched cohort analysis $(\mathrm{p}>0.4)$.

Conclusions: Within the context of an on-going debate on the consequences of incidental durotomy, we found no difference in neurologic symptoms, infection, reoperation, back pain, leg pain, or functional disability over a two-year follow up period.

230. Prospective Evaluation of a Low Dose Radiation Protocol for Minimally Invasive Transforaminal Lumbar Interbody Fusions

Justin C. Clark MD (Barrow Neurological Institute), Luis M. Tumialan MD (Barrow Neurosurgical Associates), Laura A. Snyder MD (Barrow Neurological Institute), Gary Jasmer, Frederick F. Marciano MD (Barrow Neurosurgical Associates)

Introduction: In order to address concerns with radiation dose in minimally invasive transforaminal lumbar interbody fusions (MIS TLIF), the have previously published a retrospective analysis of a low dose protocol. The results of this protocol reduced fluoroscopy times and radiation doses dramatically in comparison to the data in the current literature. A prospective analysis of this protocol is indicated.

Methods: A prospective study of the previously reported low dose radiation protocol for MIS TLIFs. Total fluoroscopy time, radiation dose, accuracy of pedicle screw placement and operative times were prospectively analyzed on patients that underwent single level MIS TLIFs.

Results: Thirty-four consectuive patients that underwent a singlelevel MIS TLIF were prospectively followed. One hundred and thirty-six pedicle screws were placed and 43 interbody spacers inserted. There were 21 women and 13 men with an average age of 63.2 years (46-82). The mean body mass index (BMI) was 25.4 (18-36.2) Indications for surgery included spondylolisthesis $(20$ patients), degenerative disc disease with radiculopathy (12 patients) and recurrent disc herniation (2 patients). Operative levels included: 5 at L3-4, 24 at L4-5, and 5 at L5-S1. The mean operative time was 178.7 minutes (119-247). The mean fluoroscopic time was 12.14 seconds (5-24). The mean radiation dose was $0.198 \mathrm{mGy} * \mathrm{~m} 2$ (0.092-0.314). There was no revision surgery required for any of the patients in this series.

Conclusions: The combination of low dose pulse images or digital spot images in a low dose protocol decreases fluoroscopy times and radiation doses in MIS TLIFs without compromising visualization of the bony anatomy or the efficiency of the procedure. Five seconds of fluoroscopy appears to be the minimum limit for an MIS TLIF. Further evaluation of this low dose protocol in multiple centers would be of value.

\section{Diffusion Tensor Imaging is a Biomarker for Disease Severity in Cervical Spondylotic Myelopathy}

Michael Mumert MD (Medical College of Wisconsin), Aditya Vedantam MD (Medical College of Wisconsin), Michael Jirjis BS, Shekar N. Kurpad MD PhD (Medical College of Wisconsin), Marjorie C. Wang MD MPH (Medical College of Wisconsin)

Introduction: Cervical spondylotic myelopathy (CSM) is a slowly progressive disease that is multifactorial in origin. Several recent publications suggest diffusion tensor imaging, specifically fractional anisotropy (FA), may provide a biomarker for disease progression. 


\section{Meeting Abstracts: AANS/CNS Section on Disorders of the Spine and Peripheral Nerves}

We hypothesized that lower preoperative FA levels would correlate with worse preoperative function.

Methods: We prospectively studied cervical spondylotic myelopathy patients who underwent pre-surgical cervical spine DTI at our institution. FA of the level of maximal compression and mean diffusivity (MD) were calculated. MJOA, Neck disability index (NDI), and SF-36 physical component scores (PCS) were obtained in the before surgery. We used Spearman's rank to test the correlation between FA and MD with mJOA, NDI, and SF-36 PCS. Linear regression modeling was used to further test this association, adjusting for age, duration of symptoms, and T2-weighted signal abnormality.

Results: Twenty-nine subjects (17 women (58\%), mean age $55+/$ - 10) underwent cervical spine DTI. Eleven patients (38\%) had symptom duration of less than 9 months. Fifteen patients (52\%) had abnormal T2 hyperintensity within the cervical spine on preoperative MRI. The mean preoperative mJOA was $13.5+/-2.2$, NDI: 35.6+/-18.1, SF-36 PCS: 34.0+/-10.7. DTI FA significantly correlated with mJOA (rho $0.62, \mathrm{p}=<0.01$ ). FA also correlated with preoperative NDI score, but this did not reach statistical significance (rho $=0.33, \mathrm{p}=0.08$ ). No significant correlation was seen between FA and SF-36 PCS, or between MD and mJOA, NDI, or SF-36 PCS. In linear regression modeling, FA was significantly correlated with preoperative mJOA score even after taking into account age, duration of symptoms, and T2-weighted signal abnormality.

Conclusions: In patients with CSM, DTI FA at the level of maximal compression is strongly associated with preoperative mJOA score, even after taking into account age, duration of symptoms, and T2-weighted signal abnormality. Fractional anisotropy is a potential biomarker for disease severity in patients with cervical spondylotic myelopathy.

\section{ALL Release by Lateral Approach for Anterior Column} Reconstruction - Anatomy, Technique and Early Results

Luiz Pimenta MD (Instituto de Patologia da Coluna), Luis Marchi MSc (Instituto de Patologia da Coluna), Rodrigo Augusto Amaral $M D$, Thiago Coutinho MD (IPC), Rubens Jensen PhD (IPC), Leonardo Oliveira BSc (Rua vergueiro, 1421 Sala 305)

Introduction: Flat back deformity and sagittal imbalance causes important clinical symptoms. Posterior-based procedures, as osteotomies, are related to important morbidity and risks. Anterior column reconstruction though lateral access is an option to restore distal lordosis and restore sagittal balance. The objective of this study was to study the anatomy related to the access and to the ALL, and show intraop and perioperative results.

Methods: Radiological study with seventy-four patients and surgeries (14 surgeries cases, with 22 spine levels, range T12-L5) done through the retroperitoneal space and through psoas muscle, using continuous EMG guidance, discectomy, ALL release and hyperlordotic cage insertion.

Results: Disc AP diameter were greater than its adjacent vertebral bodies $(\mathrm{p}<0.001)$. The aortic bifurcation occurred at L4 (61\%); L3-4(19\%) and L4-5(17\%). The iliac veins confluence occurred at L4-5(44\%), L5 (33\%), and L4 (17\%). and this reflects the closer distance of vessels to the discs over to the vertebral bodies $(\mathrm{p}<0.001)$. The areolar space to the iliac vein increases in the cranially $(\mathrm{p}<0.001)$, while to the aorta it remains the same $(\mathrm{p}=0.7)$. After standard discectomy, the intradiscal protection was removed and repositioned anteriorly to the ALL/anterior annulus and just posteriorly to the great vessels. To perform this maneuver, it is safer to insert the protector anteriorly to the ALL/anterior annulus in the adjacent vertebral body space and then go to the disc space. ALL/ anterior annulus resection was performed with a special blade. Any vascular complication occurred. Partial anterior cage migration (1 case) during posterior supplementation.

Conclusions: Close relation of great vessels and the lumbar discs are found especially in the L4-L5 spine level. Anterior annulus and ALL should be retained for safe L4-L5 discectomy, but the realease of those structures can be safe if appropriate technique is adopted. Full investigation of the anatomical position of the vessels might be required before surgery is performed.

\section{Utility of Delayed Surgical Repair of Neonatal Brachial Plexus Palsy}

Zarina S Ali MD (University of Pennsylvania), Dara Bakar, Yun Li , Alex Judd, Hiren C. Patel MBBS, PhD (Stott Lane), Eric L. Zager MD (Univ. Of Pennsylvania Hospital), Gregory G. Heuer $M D$ PhD, Sherman C. Stein MD

Introduction: Neonatal brachial plexus palsy (NBPP) represents a significant health problem with potentially devastating consequences. The most common form of NBPP involves the upper trunk roots. Currently, primary surgical repair is performed if clinical improvement is lacking. There has been increasing interest in "early" surgical repair of NBPPs, occurring within 3-6 months of life. However, early treatment recommendations ignore spontaneous recovery in Erb's cases. We chose to evaluate the optimal timing of surgical repair in this group with respect to quality of life.

Methods: We formulated a decision analytic model to compare four treatment strategies (no repair, or repair at 3, 6, or 12 months of life) for infants with persistent NBPPs. The model derives data from a critical review of published studies and projects health-related quality of life and quality adjusted life years over a lifetime.

Results: When evaluating the quality of life of infants with NBPP, improved outcomes are seen with delayed surgical repair at twelve months, compared to no repair or repair at early and intermediate time points, at three and six months, respectively. ANOVA of the differences among the 4 groups is highly significant $(\mathrm{F}=8369 ; \mathrm{p}<0.0001)$. Pairwise post hoc comparisons reveal that there are highly significant differences between each pair of strategies $(\mathrm{p}<0.0001)$. Meta-regression shows no evidence of improved outcomes with more recent treatment dates, compared to older ones for either nonsurgical or for surgical treatment $(\mathrm{p}=0.767$ and 0.865 , respectively).

Conclusions: Our data support a delayed approach of primary surgical reconstruction to optimize quality of life. Early surgery for NBPPs may be an overly aggressive strategy for infants that would otherwise undergo spontaneous recovery of function by twelve months. A randomized, controlled trial would be necessary to fully elucidate the natural history of NBPP and determine the optimal time point for surgical intervention.

\section{Hemangiomas of the Spine: Results of Surgical Management and Prognostic Variables for Local Recurrence and Mortality in a Multicenter Study}

Christina L Goldstein MD FRCSC (Toronto Western Hospital), Peter Varga, Ziya L. Gokaslan MD (Johns Hopkins University), Stefano Boriani MD (Rizzoli Institute,), Alessandro Luzzati, Richard p Williams MB BS FRACS FAOrthA (BriSpine), Laurence $D$. Rhines MD (University of Texas-MD Anderson Cancer Center), Charles Fisher MD, Dean Chou M.D. (Universitty of California San Francisco), Mark B. Dekutoski MD (The Mayo Clinic), Nasir A Quraishi, Chetan Bettegowda MD (Johns Hopkins Hospital), Norio Kawahara, Michael G. Fehlings MD PhD FRCSC FACS (Toronto Western Hospital)

Introduction: Optimal surgical treatment of, and risk factors for local recurrence and mortality associated with, symptomatic spinal hemangiomas remain unclear. This multicenter cohort study aims to quantify rates of local recurrence and mortality following surgical treatment of symptomatic spinal hemangiomas and to identify prognostic variables for local disease control and death. 


\section{Meeting Abstracts: AANS/CNS Section on Disorders of the Spine and Peripheral Nerves}

Methods: AOSpine Knowledge Forum Tumor investigators created an ambispective database of surgically treated patients with symptomatic spinal hemangiomas. Patient data pertaining to demographics, clinical presentation, diagnosis, treatment, crosssectional survival, local recurrence, and perioperative morbidity were collected. Tumors were classified according to Enneking and Weinstein-Boriani-Biagini. Descriptive statistics were summarized and Kaplan-Meier curves illustrated mortality and recurrence. Fisher's exact and log-rank tests identified prognostic variables for recurrence and mortality.

Results: Between 1996 and 2012, 68 patients (mean age $=51$ years, $\mathrm{SD}=16$; 43 females and 25 males) underwent surgical treatment for a spinal hemangioma with $23 \%(\mathrm{n}=13)$ classified as benign aggressive. Epidural tumor extension was present in $55 \%$ of patients $(\mathrm{n}=33)$. Pain was a presenting symptom in $82 \%(\mathrm{n}=54), 31 \%(\mathrm{n}=20)$ had a pathologic fracture, and $37 \%(n=24)$ were neurologically compromised. Twenty-three patients $(35 \%)$ underwent preoperative embolization. Enneking appropriate surgery was performed in $79 \%$ of patients $(n=44)$. Adjuvant radiotherapy was employed in $10 \%$ of patients $(n=7)$. The local recurrence rate was $3 \%(n=2)$ with no recurrences in patients undergoing marginal or wide resection. Prior subtotal intralesional resection was performed in one of the two patients who had a local recurrence. Mortality due to spinal hemangioma was not observed during the study period (mean followup $=3.9$ years, $\mathrm{SD}=3.8$ ).

Conclusions: This is the largest multicenter surgical cohort of spinal hemangiomas. While symptomatic spinal hemangiomas frequently present with epidural disease and neurologic compromise, excellent rates of local control and long-term survival can be obtained when strict oncologic treatment principles are followed during the index surgery.

\section{Intraoperative 32P Plaque Brachytherapy in Patients with} Circumferential and Previously Radiated Spinal Metastases

Ilya Laufer MD (Memorial Sloan-Kettering Cancer Center), Aaron Bradley Chance (The University of Texas Medical School), Michael $R$ Folkert MD PhD, J. Bryan Iorgulescu BS, Eric Lis, Yoshiya Josh Yamada MD, FRCP (Memorial Sloan Kettering Cancer Center), Mark H. Bilsky MD (Memorial Sloan Kettering Cancer Center)

Introduction: Separation surgery followed by stereotactic radiosurgery (SRS) has been shown to provide safe and durable tumor control in patients with spinal metastases. Patients with metastases encircling the spinal cord provide a difficult challenge for postoperative SRS since the contours must cover the entire preoperative tumor volume, including the dural margin, without risking spinal cord toxicity. The dosimetry is even more difficult in patients with previously radiated circumferential tumors. A 32P brachytherapy plaque was developed in order to provide a cytotoxic radiation dose to the dural margin intraoperatively and to facilitate post-operative SRS.

Methods: The study included patients who underwent separation surgery for spinal metastases between 2002 and 2013. The plaque became available in 2009. Since the plaque requires a few days of preparation, not all patients who would have benefitted from the plaque were treated due to the occasional urgent nature of surgery. The treatment group included 69 patients who underwent intraoperative $32 \mathrm{P}$ brachytherapy. The 62 patients in the control group had circumferential epidural tumor, but underwent separation surgery without brachytherapy as a $32 \mathrm{P}$ plaques was unavailable. Charts and imaging were reviewed to determine tumor histology, radiation history, surgical complications and tumor control.

Results: The control population included $47 \%$ previously radiated and $36 \%$ radiosensitive tumors. The brachytherapy population included $88 \%$ previously radiated and $13 \%$ radiosensitive tumors. All of the control patients and only 59\% of the brachytherapy patients underwent post-operative RT. The recurrence rate of $28 \%$ was noted in the control group (median follow-up 6.3 months) and $19 \%(\mathrm{p}<0.14)$ in the brachytherapy group (median follow-up 7.6 months). Complications occurred in $8 \%$ of the control and in $10 \%$ of the brachytherapy patients.

Conclusions: $32 \mathrm{P}$ plaque improves local control in patients with circumferential spinal metastases and facilitates post-operative SRS without an increase in surgical complications.

236. START Study: Sacroiliac JoinT ARThrodesis: A Retrospective Outcomes Study of the First 56 Minimally Invasive Fusions

Stephanie Falatko (Detroit Medical Center), Pradeep Setty D.O. (St. John Providence Health System/Michigan State University), Kristophe J Karami DO MS (Johns Hopkins University, Neurosurgery/Spine Center), Daniel Carr, Teck Mun Soo

Introduction: Advances in minimally invasive sacroiliac joint (SIJ) fusion have emerged in recent years to address dysfunction refractory to conservative treatment. Limited data is available to correlate fusion with improved patient outcomes. Our goal is to demonstrate improvement in pain scores, disease burden and disability by validated outcomes questionnaires following arthrodesis.

Methods: Patients with suspected SIJ dysfunction underwent two SI joint blocks. In those with improvement in pain severity, minimally invasive SIJ arthrodesis was performed using a series of three porous, plasma coated titanium implants (iFuse, SI-Bone Inc, San Jose, CA). Charts were retrospectively reviewed from July 2011 through December 2012, and 56 fusions were identified that met inclusion criteria. Primary outcome measures including the Visual Analog Pain Scale, Short Form (SF)-12, Oswestry Disability Index and patient satisfaction were evaluated pre-operatively and at 12 months. Secondary measures including operative time, blood loss and length of stay (LOS) were also analyzed. Non-parametric tests using SPSS software determined if pain, disease burden and disability improved significantly over time.

Results: Demographics revealed female prevalence (69.6\%) with a mean age of 55 years. Significant improvement from preoperative pain scores was seen at one year for both back and leg pain, $\mathrm{p}=0.000$. The Oswestry Disability Index and SF-12 physical also reached statistical significance, $\mathrm{p}=0.000$, at one year. The only parameter that approached, but did not reach significance was the SF-12 mental, $p=0.084$. Finally, operative time, blood loss and LOS were 50 minutes, $<50 \mathrm{cc}$ and approximately 1.1 days, respectively. Overall, $70 \%$ of patients were satisfied with their outcome and surgical experience.

Conclusions: Minimally invasive sacroiliac joint fusion is an effective means to address SIJ dysfunction. Patients clearly improve over time and are satisfied with both outcome and surgical experience.

\section{Clinical Outcome of Patients with Lumbar Fusion Using the Cortical Bone Trajectory}

Matthew Neal MD (Wake Forest University Baptist Medical Center), Analiz Rodriguez MD PhD (Wake Forest University School of Medicine), Aravind Somasundaram , Ann Liu B.S., Wesley Hsu MD (Wake Forest School of Medicine), Charles L. Branch MD (Wake Forest Baptist Health)

Introduction: Traditional pedicle screw fixation involves a transpedicular path along the anatomic axis of the pedicle. This trajectory remains the gold standard used in spinal fusion techniques. The cortical bone trajectory (CBT) follows a caudocephalad path and a laterally directed path in the transverse plane which allows for increased interface of the screw with cortical bone. We herein describe our clinical series of patients who underwent lumbar spinal 


\section{Meeting Abstracts: AANS/CNS Section on Disorders of the Spine and Peripheral Nerves}

fusion using CBT. To our knowledge, this is the first study to review clinical outcomes in a patient cohort who underwent lumbar fusion using CBT.

Methods: 56 consecutive patients who underwent de novo lumbar spinal fusion with CBT and posterior interbody grafting were reviewed retrospectively. Basic demographics as well as symptom/ pain improvement and Lenke fusion grade were obtained.

Results: Average age was 61 (range 29-88) with $48 \%$ female and $52 \%$ males. All but one patient underwent MAST® (minimal access spinal technologies) MIDLF technique. Intra-operative imaging was used in all cases (54 C-arm, 2 O-arm). L4-5 was the most common interspace fused (39\%). Average hospital length of stay and follow up imaging interval were 2.47 days and 8 months, respectively. Average clinical follow-up was 17 months (range: 6-25months) with $80 \%$ of patients having greater than a year follow-up. By the time of presentation, all patients will have a year of clinical follow-up. 88\% of patients had improvement of pain and symptoms at last outpatient follow-up. Radiographic imaging showed that $96 \%$ of patients had Lenke fusion grades of A or B (Class A: 31, Class B:23). One patient had Class $\mathrm{C}$ fusion grade and another patient did not have radiographic follow-up. 2 patients had complications which required surgical revision for screw repositioning. No patient had a major complication related to the surgery.

Conclusions: Patients who underwent lumbar fusion using CBT had improved pain and symptoms on follow-up as well as high fusion rates radiographically. Therefore, the utilization of CBT in lumbar fusion shows promise as an alternative to traditional pedicle screw trajectory.

238. Cost Comparison of Intraoperative 3-Dimensional Imaging versus Electrophysiologic Monitoring to Check Pedicle Screw Accuracy

Christopher Villar (University of Illinois at Peoria), Daniel Robert Fassett MD (Illinois Neurological Institute)

Introduction: Accuracy of pedicle screw placement can be evaluated intraoperatively with2-D imaging, electrophysiologic stimulation (ES) of the screws, and new 3-D imaging systems. Determining screw accuracy can be unreliable with 2-D imaging. ES of pedicle screws may help insure accuracy but can add significant costs and are not $100 \%$ reliable. Intraoperative 3-D imaging (Intraop3D) can provide high quality images to evaluate screw accuracy but require significant capital expenditures. The purpose of this study was to compare the cost of ES to evaluate screw accuracy with the cost of Intraop3D.

Methods: A cost analysis was performed at a high-volume tertiary care center in the Midwest. An O-arm acquired from Medtronic , Inc. had costs calculated for an annual basis. Neurological monitoring was routinely performed to check pedicle screw accuracy at this facility with a contract from an outside vendor to provide this service.

Results: Much of the costs of Intraop3D are fixed costs based upon initial equipment expenditure and annual service cost. The only variable cost is the technologist needed to run the equipment. Depreciation costs for the O-arm are $\$ 150,000, \$ 121,000$, and $\$ 97,000$ in the first three years after purchase for an average annual cost of $\$ 122,000$. Annual service agreement costs are $\$ 20,000$ thus giving a total fixed cost of $\$ 142,000$ annually for the O-arm. The only variable cost is $\$ 23.45 / \mathrm{hr}$ for the technician which is negligble. The average cost for monitoring was $\$ 1,100$ per case. If the $\mathrm{O}$-arm was used for the sole purpose of checking pedicle screw instrumentation, the cost per case would be depend upon case volume. A break even case volume for the O-arm in comparison to electrophysiological monitoring would be 129 cases $(\$ 142,000 / \$ 1,100)$.

Conclusions: Intraop3D systems are costly but at high-volume centers may be a more accurate and cost effective alternative to electrophysiological monitoring to check accuracy of pedicle screw instrumentation.

\section{The Impact of Preoperative Depression on Quality of Life Outcomes Following Lumbar Surgery}

Jacob A Miller BS (Cleveland Clinic Lerner College of Medicine), Thomas Mroz, Adeeb Derakhshan BS, Matthew D. Alvin MBA MA (Case Western Reserve Unviersity School of Medicine), Daniel Lubelski ,Edward C. Benzel MD (Cleveland Clinic Foundation)

Introduction: Lumbar surgery has been demonstrated to significantly improve patient quality of life (QOL). However, in light of pressures to reduce costs and improve outcomes following treatment, many studies have investigated the effect of preoperative risk factors upon outcomes. Though prior studies have identified depression as a predictor of diminished improvement, none have utilized the Patient Health Questionnaire-9 (PHQ-9), the gold standard in assessing depression as based upon DSM-IV criteria. In this study, we assessed the impact of preoperative depression and patient characteristics on QOL outcomes after lumbar surgery. We hypothesized that lumbar surgery significantly improves outcomes in all patients with diminished improvement among the preoperatively depressed.

Methods: Health Status Measures (HSM) data was prospectively collected from 2008 through 2012. Patients undergoing lumbar surgery with followup greater than 6 months were included in the study, while those younger than 18 years were excluded. Demographic, operative, comorbidity, and HSM data were collected at outpatient visits. HSM data included the EQ-5D (Euroqol 5-Dimensions), PDQ (Pain Disability Questionnaire), and PHQ-9 surveys. Simple and multiple linear regression was performed with all variables using EQ-5D quality-adjusted life years (QALY) as the dependent variable.

Results: Of 3823 eligible patients, 919 had followup greater than 6 months and were older than 18 years. The average followup was $421 \pm 217$ days. $179(19.5 \%)$ were identified as moderately or severely depressed by the PHQ-9. The cohort demonstrated improvement postoperatively in the EQ-5D, PDQ, and PHQ-9. Multiple linear regression identified increasing pain (PDQ, $\beta=-0.0017, \mathrm{p}=0.0009$ ) and depression (PHQ-9, $\beta=-0.0044, \mathrm{p}<0.0359$ ) as predictive of diminished QALY improvement when adjusting for all other variables.

Conclusions: This study represents the largest investigation in preoperative depression and quality of life outcomes following lumbar surgery. Furthermore, its prospective nature and utilization of the PHQ-9 strongly support the assessment of preoperative depression when selecting patients for surgical management.

\section{Neurosurgery Skills Training - Past, Present and Future:} Hands-on Skills Training Modules to Virtual Reality Simulation

Ashish Suri MD (All India Institute of Medical Sciences), Payal Jotwani BS (University of Central Florida), Britty Baby, Vinkle Srivastav, Tara S. Roy, Sanjeev Lalwani MBBS MD (AIIMS), Martin Bettag, Christoph Busert, Marcus Mehlitz, Subhashish Banerji, Sanjiva Prasad, Prem Kalra, Subodh Kumar, Kolin Paul, Bhawani S. Sharma MCh (Room 720, 7th floor)

Introduction: Teaching, assessment and practicing of basic neurosurgical skills via the apprenticeship-model in the operating room, is no longer considered appropriate. Issues of minimal access surgery, patient safety and cost effectiveness involve alternative Methods: of instruction.

Methods: Neurosurgery Education and Training School was established with the goal to enhance neurosurgery skills training and provide technological solutions for evaluation. The threepillared ventures of the school are: i. Hands-on Skills Training: Neurosurgery Skills Training Facility (NSTF) Neurosurgical skills training imparted is broadly covered under 5-modules: Neuroanatomy tutorial, Neuroendoscopy, Microneurosurgery, High speed drilling, Spinal instrumentation. The training is provided on syn- 


\section{Meeting Abstracts: AANS/CNS Section on Disorders of the Spine and Peripheral Nerves}

thetic, semi-synthetic models, cadavers and animals with highend equipment in an operation-room-like-environment. The skills training curriculum is defined by guidelines based on the evaluation of trainee by senior faculty and by computerized evaluation system. ii. E-Learning: The virtual education material on open source free access interactive E-learning platform and discussion forum, in the form of video library and webinars. iii. Development of Computerized Virtual Reality (VR) Systems: Bridge the gap between skills training on subjects and live operations, while supplementing the use of live tissue for skills training.

Results: Hands-on skills training: Total Daily Skills Training Sessions: 3056 Quarterly/Advanced Workshops: 18 E-learning: 483 visits/ month with $59.6 \%$ returning visits and $40.40 \%$ new visits on the NETS website from over 58 nations. 55,012 Views with 141 subscribers for neurosurgery operation, animation and graphics based videos. Average reach of data on Discussion Forum: $84.58 \%$ of the members, i.e. $46534.6 \%$ used the electronic tablets followed by $13.5 \%$ fablets. The objective versus subjective assessment was analyzed; the computerized evaluation scored above the subjective assessment in terms of unbiased independent grading.

Conclusions: The incorporation of virtual and hands-on training with parallel assessment supplements education and skills training in Neurosurgery.

241. Does the Presence of Low Back Pain Modify the Surgical Prognosis of Discectomy in Patients with Symptomatic and Refractory Lumbar Disc Herniation?

Asdrubal Falavigna PhD (Leonilda Fassoli Zatti 201/01), Orlando Righesso, Alisson Teles, Pedro Guaresi Silva

Introduction: Improving patient selection will significantly improve discectomy-related outcomes and reduce medical costs. The objective of this study was to assess if the presence of low back pain (LBP) in lumbar disc herniation (LDH) patients preoperatively was a prognostic variable, through a cohort prospective study in LDH patients refractory to conservative treatment and with symptoms of radicular pain associated or not with LBP, in whom discectomy was performed.

Methods: A one-year prospective study with 104 patients submitted to open discectomy due to $\mathrm{LDH}$. Inclusion criteria were the presence of radicular pain due to $\mathrm{LDH}$ diagnosed by magnetic resonance imaging refractory to clinical treatment. Exclusion criteria were radiological evidence of instability or not accepting to participate in the study. In the preoperative evaluation, patients were asked about the presence of LBP at least 6 months before the present sciatica crisis. The cohort was evaluated during the preoperative period, 1 month, 6 months and 1 year postoperatively with validated instruments (Oswestry Disability Index, SF-36, Beck depression inventory, Hospital Anxiety and Depression Scale, Numerical Rating Scale of Pain). Back and leg pain were assessed with NRS of pain.

Results: More than half of the patients reported LBP before the leg pain $(62 / 104 ; 59.6 \%)$. Mean axial pain in the preoperative period was $4.21(+-2.47)$ in these patients compared with 2.09 $(+-1.87)$ in patients without a history of LBP $(\mathrm{P}<0.0001)$. After open discectomy, improvements in leg and back pain were observed in the two groups (mean difference pre-treatment - 1 year for LBP: -2.27 +- 2.38; CI95\%: $-1.81--2.74$; P < 0.0001). Comparing patients with and without complaints of LBP 6 months previously to the radicular symptoms, no difference was observed regarding improvements in health-related quality of life measures or satisfaction rates with treatment.

Conclusions: The LBP was observed in $59.6 \%$ of the patients at least 6 months before to the radicular pain symptoms of LDH. Fortunately, the preoperative LBP improves after LDH surgical removal and was not associated with dissatisfaction or with a decrease in health-related quality of life at 1 year follow-up.
242. Does Minimally Invasive Posterior Instrumentation (PPI) Prevent Proximal Junctional Kyphosis (PJK) in Adult Spinal Deformity (ASD) Surgery? A Prospectively Acquired Propensity Matched Cohort Analysis

Praveen V. Mummaneni MD (University of California San Francisco Spine Center), Michael Y. Wang MD FACS (University of Miami), Virginie Lafage PhD (NYU Langone Medical Center), Kai-Ming G. Fu MD PhD (Weill Cornell Medical College), Jamie Terran BS, David O. Okonkwo MD PhD (University of Pittsburgh), Juan S. Uribe MD (University of South Florida Division of Neurosurgery), Neel Anand MD, Richard G. Fessler MD (Rush University Medical Center), Adam S. Kanter MD (University of Pittsburgh Medical Center Presbyterian), Frank La Marca MD (University of Michigan), Christopher I. Shaffrey MD FACS (University of Virginia), Vedat Deviren MD (Univeristy of California San Francisco), Frank Schwab MD, PhD, Shay Bess MD (Rocky Mountain Scoliosis and Spine Center), Gregory Mundis MD (San Diego Center for Spinal Disorders), International Spine Study Group

Introduction: Proximal junctional kyphosis (PJK) is an unwanted complication of ASD surgery. Multiple theories exist why PJK occurs. One potential contributor is damage to the paraspinal musculature and intervertebral stabilizers frequently disrupted during exposure and screw placement. This study aims to investigate the effect of PPI vs. Open screw placement on PJK development.

Methods: 280 pts in 2 prospective databases (MIS $\mathrm{n}=85$; OPEN $n=195$ ) were retrospectively reviewed, divided in 2 separate approaches and propensity matched for pelvic incidence - lumbar lordosis (PI-LL) and change of LL. Inclusion criteria: age $>45$, Cobb $>20^{\circ}$, min 1 yr follow up. Groups defined as: 1)cMIS- lateral interbody fusion (LIF) with PPI ( $\mathrm{n}=31)$ and 2)Hybrid (HYB)- LIF followed by open posterior instrumentation $(n=31)$. PJK was defined as Proximal junctional angle (PJA) $>10^{\circ}$ and change post op $>10^{\circ}$.

Results: A mean of 4.1 levels were fused (range 2-6). The mean age was 64 years and mean BMI was 26.1. Mean follow-up was 27.5 months. There was no preop difference between groups for LL-PI or SVA. Both groups showed significant improvement in LL (cMIS: $33^{\circ}-41^{\circ}$; HYB: $35^{\circ}-44^{\circ}$; p $<0.001$ ) and PI-LL (cMIS: $19.7^{\circ}-$ 12.4 $4^{\circ}$ HYB: $19.6^{\circ}-7.4^{\circ}$ ) and significant difference in PT. SVA remained physiologic for cMIS $(29-26 \mathrm{~mm})$ and improved in HYB (54-31 mm; $\mathrm{p}=0.024)$. The cMIS group had a smaller change in PJA $\left(+1.3^{\circ}\right)$ than HYB $\left(+6^{\circ}\right)(\mathrm{p}=0.005)$. PJK developed in $19.4 \%$ of HYB patients and $0 \%$ in cMIS $(\mathrm{p}<0.01)$. One patient in the Hybrid group required vertebroplasty for PJK. Both groups saw significant improvement in ODI (cMIS 39 to 20.1; HYB 46.7 to 30 ; $\mathrm{p}<0.001$ ).

Conclusions: The addition of PPI seems to have a protective effect on the development of PJK. The analysis controlled for preoperative sagittal alignment as well as for correction of PI-LL. HYB was effective in restoring sagittal global alignment and cMIS in maintaining it.

\section{Microendoscopic Decompression for Cervical Spondylotic Myelopathy}

Nader S. Dahdaleh MD (Northwestern University), Zachary Adam Smith MD (Northwestern Dept. of Neurological Surgery), Laura A. Snyder MD (Barrow Neurological Institute), Albert Wong MD (Northwestern University), Ricky $H$ Wong MD (University of Chicago), Sandi Lam MD (Texas Children's Hospital/Baylor College of Medicine), Richard G. Fessler MD (Rush University Medical Center)

Introduction: Cervical spondylotic Myelopathy is a common cervical degenerative disease that affects the elderly population. Spinal cord decompression is achieved through various anterior and posterior approaches including anterior cervical decompression 


\section{Meeting Abstracts: AANS/CNS Section on Disorders of the Spine and Peripheral Nerves}

and fusion, laminectomy, laminoplasty, and combined approaches. Minimally invasive endoscopically assisted decompression (MEDS) is another option that obviates muscle dissection and disruption of the posterior tension band; a cause of post laminectomy kyphosis.

Methods: A retrospective study was conducted on 10 patients with CSM who underwent MEDS between January 2002 and July 2012. Demographics, preoperative and postoperative Nurick scores and Odom scores were collected as well as preoperative and postoperative Cobbs angles

Results: The average age was 67 years (+/- 7.7), eight patients were males. The average number of disk levels operated was 2.2 (range 1 to 4$)$. The mean Nurick score was $1.6(+/-0.7)$ preoperatively and improved to $0.3(+/-0.7)$ postoperatively $(\mathrm{p}<0.0005)$. The postoperative Odom score was excellent for four patients, good for three patients, two had fair outcomes and one had a poor outcome. The average preoperative focal Cobbs angle at the levels operated was $-0.43(+/-1.9)$. The Cobbs angle at the last follow up was $0.25(+/-1.6)(\mathrm{p}=0.6)$. The average follow up was 18.9 months $(+/-32.1)$. There were no intraoperative or postoperative complications.

Conclusions: MEDS can be used as an alternative method to open laminectomy and laminoplasty in selected patients with CSM who have primarily posterior pathology and acceptable preoperative lordosis.

244. Costs of Cervical Disc Replacement versus Anterior Cervical Discectomy and Fusion for Treatment of SingleLevel Cervical Disc Disease: An Analysis of the Blue Health Intelligence Database for Acute and Long-Term Costs and Complications

Jack E. Zigler MD, Kris Radcliff MD, Jeffrey Zigler JD, Donna D. Ohnmeiss Dr.Med.

Introduction: The purpose of this study was to compare reoperation rates, adverse event rate, as well as direct and follow-on costs of cervical disc arthroplasty (CDA) with anterior cervical discectomy and fusion $(\mathrm{ACDF})$ in patients with single-level symptomatic cervical disc disease.

Methods: This was a retrospective, matched cohort analysis of prospectively collected insurance industry allowed amounts (costs) and outcomes for patients age 18-60, who were continuously enrolled in one of 18 BlueCross BlueShield plans contributing data to the Blue Health Intelligence (BHI) claims database. Included were all patients who were treated surgically with CDA or ACDF between January 2008 and December 2009 for single-level cervical pathology, who had preoperative claims for at least six weeks of nonsurgical care, and without claims history of prior surgery.

Results: There were 6,635 ACDF patients and $327 \mathrm{CDA}$ patients who met inclusion criteria. There were no statistically significant differences in comorbidities $(\mathrm{p}=0.0884)$ or mean follow-up (ACDF 25.7 vs. CDA 26.1 months; $p=0.7140$ ). There were no statistically significant differences in pain events $(\mathrm{p}=0.17)$ or readmission rates between groups $(\mathrm{p}>0.05)$. By 36 months postoperatively, the reoperation rate was significantly greater for $\mathrm{ACDF}(10.5 \%)$ versus CDA $(5.7 \%)$ (Hazard ratio, $\mathrm{p}=0.0214)$. The index surgery and global 90-day window costs (including payment to all relevant healthcare providers and facilities, except drug costs) were significantly lower for CDA (CDA $\$ 22,761$ versus ACDF $\$ 25,029$, p=0.0086). At two years follow-up, there was a statistically significantly lower total costs paid for CDA versus ACDF (CDA \$34,979 versus ACDF $\$ 39,820)$ and cost per person per month at 1 (CDA $\$ 3,071$ versus $\mathrm{ACDF} \$ 3,634, \mathrm{p}=0.0020), 2$ (CDA $\$ 2,414$ versus $\$ 2,984$ $\mathrm{p}=0.0008$ ), and 3 years follow-up (CDA $\$ 2,291$ versus ACDF $\$ 2,874, \mathrm{p}=0.0008$ ).

Conclusions: Patients who underwent CDA for single-level degenerative disease had lower readmission rates, lower reoperation rates, and reduced index and total costs compared to ACDF patients.

\author{
245. Total Hospital Costs of Surgical Treatment for Adult \\ Spinal Deformity (ASD): An Extended Follow-up Study
}

Ian McCarthy PhD (4708 Alliance Blvd.), Michael F. OBrien MD (Miami Children's Hospital), Christopher P. Ames MD (University of California San Francisco Neuro Surgery), Han Jo Kim MD, Justin S. Smith MD PhD (University of Virginia Health System), Oheneba Boachie-Adjei MD, Frank Schwab MD, PhD, Eric Klineberg MD (University of California - Davis), Christopher I. Shaffrey MD FACS (University of Virginia), Munish Gupta MD (University of California - Davis; Ortho Surgery), Richard A. Hostin MD, International Spine Study Group

Introduction: While the costs of primary surgery, revisions, and selected complications for ASD have been individually reported in the literature, total costs over several years following surgery have not been assessed. Understanding the total per-patient cost of surgical care is critical in accurately measuring the cost-effectiveness of surgical treatment for ASD.

Methods: Single-center, retrospective analysis of 523 consecutive patients undergoing surgical treatment for ASD prior to January 2011. Costs were collected from hospital administrative data on the total hospital costs incurred for the operation and any related readmissions, expressed in 2010 dollars and discounted at $7 \%$ per year. Comparative costs on total hip replacement (THR) and CABG were collected from the literature.

Results: Patients were predominantly female ( $\mathrm{n}=446$, or $85 \%)$ with an average age of 49 (18 to 82) and average follow-up of 4.6 years (2 to 8). Total hospital costs averaged $\$ 117,876(\mathrm{std}=\$ 60,550)$, with primary surgery averaging $\$ 103,766(\mathrm{std}=\$ 41,707)$ and total readmission costs averaging $\$ 70,722$ per patient $(\mathrm{std}=\$ 64,320$, $\mathrm{n}=145$, or $28 \%$ of all patients). Average costs across all patients significantly increased $(\mathrm{p}<0.03)$ after primary surgery, from $\$ 110,282$ at one-year follow-up to $\$ 122,077$ at five-year follow-up. Perpatient costs did not significantly increase in successive follow-up years beyond five years $(\mathrm{p}>0.05)$.

Conclusions: Per-patient costs were similar to previously reported long-term costs for CABG and five times higher than that of THR, although the relative cost of revision compared to primary surgery was lower for ASD than for THR. The incidence of readmissions increased the average cost of ASD surgery by more than $170 \%$, illustrating the financial burden of revisions/re-operations; however, the cost-burden resulting from readmissions was relatively acute, tapering off within 5 years following surgery. This has important implications for the lifetime cost-effectiveness of ASD surgery, as benefits of surgery likely extend beyond 5 years while average per-patient costs remain steady.

\section{Understanding the Effect of Surgical Complication on the Value of Surgical Spine Care: Evolution of the Healthcare Value Equation}

Saniya S. Godil MD (Vanderbilt University), Scott L. Zuckerman MD (Vanderbilt University School of Medicine), Matthew J. McGirt $M D$

Introduction: Surgical treatments of structural low back diseases are facing increasing scrutiny. Complications are an inevitable part of any surgery. A recent study demonstrated that complications do not affect outcomes after lumbar fusion; however, no studies have related the impact of complications on the value of spine surgery. Therefore, we set out to determine the impact of complications on effectiveness, cost and value of spine surgery.

Methods: All patients undergoing elective lumbar surgery for degenerative spine disease were enrolled into a prospective registry. Data was collected on patient demographics, treatment variables and complications. Patient reported outcomes (PROs) and medical resource utilization were prospectively recorded at 


\section{Meeting Abstracts: AANS/CNS Section on Disorders of the Spine and Peripheral Nerves}

baseline and 1-year. Surgical DRG/CPT based medicare fees and medical resource utilization were used to estimate direct healthcare costs. Patient and caregiver work-day losses were used to calculate indirect costs. Value of lumbar surgery was calculated as cost per QALY gained.

Results: A total of 528 patients were included [Major complication $=67$ patients; No complication $=461]$.The complications group had significantly higher comorbidities $(\mathrm{p}=0.02)$, worse ASA grade $(\mathrm{p}=0.04)$, and higher proportion of patients underwent revision surgery $(\mathrm{p}=0.02)$. After adjusting for demographics and treatment variables, there was no significant difference in 1-year outcomes in the two groups: NRS-BP ( $\mathrm{p}=0.96)$, NRS-LP $(\mathrm{p}=0.26)$, ODI $(\mathrm{p}=0.70)$, SF-12 PCS $(p=0.41)$, SF-12 MCS $(p=0.44)$ and EQ-5D $(p=0.73)$. Fewer patients returned to work in complications group (82.6\% vs. $87.7 \%$ ) and there was a delay in median time to return to work (50 days vs. 40 days). $\operatorname{Direct}(\$ 48,496$ vs. $\$ 22,579)$ and indirect $(\$ 6,342$ vs. $\$ 4,892)$ healthcare cost was significantly higher in complications group leading to significant reduction the value of lumbar spine surgery from $\$ 62,436 / \mathrm{QALY}$ gained to $\$ 152,330 / \mathrm{QALY}$ gained.

Conclusions: In our real-world prospective cohort study, major complications did not affect 1-yr outcomes after lumbar spine surgery. However, complications significantly increased healthcare cost and delayed return to work after surgery, reducing the value of spine surgery from $\$ 62,436 /$ QALY gained to $\$ 152,330 /$ QALY gained.

\section{Cost-effectiveness of Minimally Invasive Versus Open} Correction of Adult Degenerative Deformity

Raqeeb M. Haque MD (Cleveland Clinic Foundation), Omar Minhaj Uddin BA, Ross York-Erwin , Joel M. Press MD, Patrick Alexander Sugrue MD (Washington University School of Medicine), Jessica A Drope , Sara Thompson, Yousef Ahmed, Tyler R. Koski MD (Northwestern University), Richard G. Fessler MD (Rush University Medical Center)

Introduction: Back pain is an increasing concern for the aging population. This study aims to evaluate if minimally invasive surgery presents cost-effective attributes compared to open surgery in treating adult degenerative deformity.

Methods: Seventy-one patients with adult degenerative scoliosis received two-stage, multilevel surgical correction through either an MIS approach with posterior instrumentation $(n=38)$ or an open midline approach $(n=33)$. Costs were derived from hospital and rehabilitation charges. Length of stay, blood loss, and radiographic outcomes were obtained from electronic medical records. Functional outcomes were measured with Oswestry Disability Index (ODI) and Visual Analogue Scale (VAS) surveys.

Results: Patients in both cohorts were similar in age (AgeMIS=65.68, AgeOpen=63.58, p=0.28). Mean follow-up was 18.16 months and 21.82 months for the MIS and Open cohorts $(\mathrm{p}=0.34)$. MIS and Open cohorts had an average of 4.37 and 7.61 levels of fusion, respectively $(\mathrm{p}<0.01)$. Total inpatient charges were lower for the MIS cohort $(\$ 269,807$ vs $\$ 391,889, \mathrm{p}<0.01)$ and outpatient rehabilitation charges were similar $(\$ 41,072$ vs $\$ 49,272$, $\mathrm{p}=0.48)$. MIS patients experienced reduced hospital stay $(6.16$ days vs 12.39 days, $\mathrm{p}<0.01)$ and blood loss $(\mathrm{EBL}-\mathrm{MIS}=470.26 \mathrm{~mL}$, EBL-Open $=2872.73 \mathrm{~mL}, \mathrm{p}<0.01)$. Baseline ODI was lower in the MIS cohort (40.03 vs. 48.04, $\mathrm{p}=0.03$ ) and both cohorts experienced similar 1-year improvement (deltaODI-MIS=-15.98, deltaODI-Open $=-21.96, \mathrm{p}=0.25)$. Baseline VAS scores were similar (VAS-MIS=6.56, VAS-Open=7.10, $\mathrm{p}=0.32$ ) but MIS patients experienced less reduction after 1 year (deltaVAS-MIS $=-3.36$, deltaVAS-Open $=-4.73, \mathrm{p}=0.04)$. Pre-operative SVA were comparable (PreopSVA-MIS=63.47 mm, PreopSVA-Open=71.3mm, p=0.60) but MIS patients had larger post-operative SVA (PostopSVAMIS $=51.17 \mathrm{~mm}$, PostopSVA-Open $=28.17 \mathrm{~mm}, \mathrm{p}=0.03$ ).

Conclusions: Minimally invasive surgery demonstrated reduced costs, blood loss, and hospital stay while open surgery exhibited greater improvement in VAS scores, deformity correction, and sagittal balance. Additional studies with more patients and longer follow-up will determine if MIS presents cost-effective benefits for certain subsets of patients with adult degenerative scoliosis.

\section{Differences in Functional Recovery in Immune Compro- mised Rats after Contusive Spinal Cord Injury}

Catherine Miller MD (University of Minnesota Medical School, Neurosurgery), Ann Margaret Parr MD PhD FRCSC (University of Minnesota)

Introduction: Spinal cord injury is a devastating problem caused by both the immediate trauma as well as from delayed secondary effects. Several mechanisms of secondary injury have been demonstrated including alterations in the blood-brain barrier, edema, metabolic disturbances, and a neuroinflammatory response. Extensive research has been done to understand and potentially protect from further neuronal damage by these mechanisms. The goal of this paper is to further explore the role of the immune system in terms of functional recovery after a contusion spinal cord injury in a rat model.

Methods: A cohort of adult female Sprague Dawley rats and athymic nude rats underwent a T8/9 laminectomy, followed by a contusion injury created by the Infinite Horizon spinal cord impactor device (Precision System and Instrumentation, Lexington, KY). The Basso-Beattie-Bresnaham locomotor rating scale was used to assess the locomotor recovery in both groups on postinjury day two and then on a weekly basis for 8 weeks after injury. Analysis of the injured spinal cord was performed to identify differences in gene expression.

Results: Despite receiving similar injuries, the nude mice showed a slightly better initial locomotive score. There was a significant difference in functional recovery with the nude rats showing a superior improvement at all-time points when compared to the Sprague Dawley rats. The results of the gene expression analysis will be discussed.

Conclusions: The role of the immune system in spinal cord injury and possible therapies to target the inflammatory response have been a topic of significant investigation. This is the first paper to describe a difference in functional recovery in a contusion injury model between nude and Sprague Dawley rats. Furthermore, this gene analysis can identify potential gene targets for therapy.

\section{Prospective Analysis of Risk Factors for Proximal Junctional Failure in Adult Deformity Patients Improve Our Understanding of Surgical Treatment Strategies for Adults with Spinal Deformity}

D. Kojo Hamilton MD (Oregon Health \& Sciences University), Robert Hart MD (Ohsu Spine Center), Jayme Hiratzka MD, Shay Bess MD (Rocky Mountain Scoliosis and Spine Center), Christopher I. Shaffrey MD FACS (University of Virginia), Christopher P. Ames MD (University of California San Francisco Neuro Surgery), Virginie Lafage PhD (NYU Langone Medical Center), Praveen V. Mummaneni MD (University of California San Francisco Spine Center), Justin S. Smith MD PhD (University of Virginia Health System), Eric Klineberg MD (University of California - Davis), Ian McCarthy PhD (4708 Alliance Blvd.), Douglas C. Burton MD, Richard A. Hostin MD, International Spine Study Group

Introduction: Proximal Junctional Failure (PJF), a severe form of Proximal Junctional Kyphosis (PJK) with evidence of mechanical failure, is an important concern in adult deformity surgery (ADS). Post-operative sagittal imbalance and proximal/distal end points for the fusion are possible risk factors for PJF. Prospective evaluation of these factors has not been reported. 


\section{Meeting Abstracts: AANS/CNS Section on Disorders of the Spine and Peripheral Nerves}

Methods: 352 ASD patients (10 centers) were followed prospectively with minimum 1-year follow-up. PJF was defined as increased proximal kyphosis of $>10^{\circ}$ plus fracture of UIV or UIV+1 or instrumentation failure. Proximal Junctional Kyphosis (PJK) was defined as increased kyphosis of $>10^{\circ}$ without mechanical failure. Patients were grouped as PJF, PJK, or neither (NoPJF). Proximal fusion levels were defined as Upper Thoracic (UT, T2-T5) or Thoracolumbar (TL, T9-T12). Age, Sacral Slope (SS), Pelvic Tilt (PT), Pelvic Incidence (PI), Sagittal Vertical Axis (SVA), Lumbar Lordosis (LL), and PI-LL were compared.

Results: There were $41(11.7 \%)$ PJF, 54 (15.3\%) PJK, and 257 (73.0\%) NoPJF patients with 1-year follow-up. There were significant differences in age (65.1 vs 55.7; $\mathrm{p}=0.001)$ and pre-op PT (26.8 vs 22.0; $\mathrm{p}=0.043$ ) between PJF and NoPJF patients, respectively. There was a trend toward increased pre-op SVA and PI-LL, as well as correction in SVA, LL, and PI-LL among PJF patients. Postop SVA and PI-LL were similar between PJF and NoPJF patients. No patient experienced PJF without fusion to the pelvis. Among patients fused to the pelvis, there was no difference between UT vs LT proximal endpoints for rate of PJF (13.4\% vs 16.8\%; $>>0.05)$, although the rate of PJK was greater for UT patients (26.8\% vs $13.5 \% ; \mathrm{p}=0.017$ ).

Conclusions: Post-op sagittal alignment and proximal fusion end point did not differ between PJF and NoPJF patients. Older patients with worse pre-op sagittal imbalance, larger sagittal corrections and pelvic fixation were identified as those at greatest risk of PJF.

250. Quantitative Analysis of MRI Spinal Cord Compression and Canal Compromise in Patients with Cervical Spondylotic Myelopathy: Results from the Prospective, Multicenter AOSpine North American Study

Aria Nouri BA MD (Toronto Western Hospital), Lindsay Tetreault Bsc (Toronto Western Hospital), Juan Jose Zamorano MD (Neurosurgery Division-Toronto Western Hospital), Kristian Dalzell, Michael G. Fehlings MD PhD FRCSC FACS (Toronto Western Hospital)

Introduction: Magnetic Resonance Imaging (MRI) is the primary imaging modality for confirming the diagnosis of cervical spondylotic myelopathy (CSM) but has been limited in its ability to stratify disease severity and predict surgical outcome. In the present study, quantitative analyses of spinal cord compression (SCordC) and modified calculations of spinal canal compromise (SCanalC) were performed and compared with outcome data at 1-year with an effort to provide prognostic guidance.

Methods: Of the 278 patients enrolled, 106 had MRIs available for analysis. T2-weighted images were reviewed by 3 investigators and the sites of maximal SCordC and SCanalC were identified. Measurements were carried out using Fehlings et al (1999) Methods:; however, the measurement limits were modified and demarcated by the posterior longitudinal ligament anteriorly and ligamentum flavum posteriorly. Univariate analyses were performed to evaluate the relationship between these measurements and outcome (mJOA). Multivariate ordinal logistic regression analysis was subsequently conducted controlling for age and baseline severity score.

Results: Average SCordC was 33.9 $\pm 15.4 \%$ (range: -4.6, 64.8\%) and $49.3 \pm 13.1 \%$ (range: $16.12,75.3 \%$ ) for SCanalC. mJOA at 1-year follow-up was trichotomized for ordinal logistic regression: excellent outcome was defined as mJOA $>=17$, good outcome as $15-16$, and poor outcome as $<15$. Univariate analysis found no significant association between $\mathrm{SCordC}$ and outcome $(\mathrm{OR}=0.99$, $95 \% \mathrm{CI}=0.96-1.01, \mathrm{p}=0.36)$. In contrast, $\mathrm{SCanalC}$ was significantly related to outcome: for every $1 \%$ decrease in SCanalC, the odds of moving from a lower to a higher outcome group increased by $4 \%(\mathrm{OR}=0.96,95 \% \mathrm{CI}=0.93-0.99, \mathrm{p}=0.011)$. Following control for important confounding variables, canal compromise was still an important predictor $(\mathrm{OR}=0.96,95 \% \mathrm{CI}=0.93-0.99, \mathrm{p}=0.024)$.
Conclusions: SCanalC is a strong predictor of outcome in CSM patients 1-year post surgery and likely represents a fundamental part of CSM pathogenesis. Why ScordC is not, remains to be investigated. Thus, SCanalC calculations should be computed in all patients and considered in surgical decision-making.

251. A Clinical Prediction Model to Assess Surgical Outcome in Patients with Cervical Spondylotic Myelopathy: Internal and External Validation using the Prospective Multicenter AOSpine North American and International Datasets in 764 Patients

Lindsay Tetreault Bsc (Toronto Western Hospital), Branko Kopjar MD, Paul M. Arnold MD (Department of Neurosurgery), Michael G. Fehlings MD PhD FRCSC FACS (Toronto Western Hospital)

Introduction: The objective of this study is to internally and externally validate a clinical prediction rule originally formulated to predict surgical outcome in patients with cervical spondylotic myelopathy (CSM). A valid model will allow for accurate quantification of patients' likely outcome and for the alignment of clinical perceptions with evidence-based reality.

Methods: A prediction model was constructed using data from 272 patients enrolled in the CSM-North American prospective study. Bootstrapping was used for internal validation. The area under the receiver operating curve (AUC) computed during bootstrapping was compared to the AUC of the original model. The original model was externally validated on a second prospective study that recruited 492 patients from 16 international sites. Discriminative performance and calibration were evaluated.

Results: The original model included age $(\mathrm{OR}=0.96)$, duration of symptoms ( $\mathrm{OR}=0.78)$, baseline mJOA $(\mathrm{OR}=1.24)$, depression or bipolar disorder $(\mathrm{OR}=0.39)$, impaired gait $(\mathrm{OR}=2.51)$ and smoking status $(\mathrm{OR}=0.44)$. The AUC for the model was $0.783(0.728-0.836)$ and across the bootstrap replicates was $0.782(95 \% \mathrm{CI}=0.777-0.786)$, reflecting good discrimination and internal validity. The model tested on the CSM-International dataset yielded a ROC curve with an area of $0.73(95 \% \mathrm{CI}=0.68-0.77)$, a calibration slope of 0.81 and a significant observed:expected ratio of 0.79 (95\% CI=0.721-0.864). The odds ratios generated for baseline mJOA, impairment of gait and age using the external dataset were very similar to the original values: $1.28,2.39$ and 0.97 , respectively. Duration of symptoms $(\mathrm{OR}=0.92)$ and smoking status $(\mathrm{OR}=0.65)$ had different odd ratios than the original model but the direction of their relationship with outcome was the same.

Conclusions: We have developed a valid clinical prediction rule based on the following key variables: baseline mJOA, age, impaired gait, smoking, duration of symptoms and psychiatric illness. Using these data, clinicians can make informed judgments on clinical care and provide appropriate advice to patients and families.

\section{Complication Avoidance and Management in Thoracic Disc Surgery: Experience with 64 Patients}

Noel I. Perin MD FRCS(Ed) (NYU Langone Medical Center, Department of Neurosurgery), Russell G. Strom MD (NYU Langone Medical Center), Vin Mathur MD (St. Louis University Hospital), Heather Givans NP

Introduction: Symptomatic thoracic disc herniation (TDH) is a rare condition with significant treatment risks. Here we review our experience for complication avoidance and management strategies.

Methods: All TDH cases by the senior author were reviewed from 2000 to 2012. The incidence, management, and natural history of complications were recorded. Complication avoidance strategies were noted. Later in the series, an anterior tubular retractor-based technique was developed to minimize complication risk (Figure 1). In this combined approach, thoracoscopy was used to safely retract 


\section{Meeting Abstracts: AANS/CNS Section on Disorders of the Spine and Peripheral Nerves}

the lung and great vessels while an expandable tubular retractor was deployed via mini-thoracotomy.

Results: 64 patients were treated for TDH through a variety of approaches. There were 15 major complications: 7 dural tears (1 requiring reoperation), 3 cases of intercostal neuralgia persisting $>$ 3 months, 1 pneumothorax, 2 cases of motor deterioration, and 2 cases of mechanical instability necessitating further surgery. Several strategies were effective at managing these complications, including routine lumbar drain placement for dural tears and radiofrequency ablation for persistent intercostal neuralgia. One case of persistent CSF leakage was successfully treated with a laparoscopicallymobilized omental flap over the dural defect (Figure 2). Besides intercostal neuralgia, there were no complications from the 13 thoracoscopy-guided tubular retractor approaches. Many strategies successfully prevented complications altogether, including routine chest tube placement for anterior approaches and preoperative Guglielmi detachable coil insertion into the associated rib head to facilitate intraoperative localization. TDH complications not occurring in this series included wrong-level surgery, hemothorax, chylothorax, pseudarthrosis, and infection.

Conclusions: TDH continues to carry significant treatment risks in the modern era. Several strategies are effective at decreasing the likelihood of complications and minimizing morbidity when they do occur. Minimally-invasive techniques appear to lower the risk of approach-related complications such as pneumothorax, persistent pain, and infection.

\section{Intraoperative Vancomycin Use in Spinal Surgery: Single Institution Experience and Microbial Trends}

George M. Ghobrial MD (Thomas Jefferson University), Vismay Thakkar, Edward Andrews , Michael J. Lang MD (Thomas Jefferson University Hospital), Ameet V. Chitale MD (Thomas Jefferson University Hospital), Mark E. Oppenlander MD (Barrow Neurological Institute), Christopher Michael Maulucci MD (University of Maryland Hospital), Joshua E. Heller MD (Thomas Jefferson University), Ashwini Dayal Sharan MD (Thomas Jefferson University), James S. Harrop MD FACS (Jefferson University Hospital), Jack Jallo MD, PhD, FACS (Thomas Jefferson University Hospital), Srinivas K. Prasad MD (Thomas Jefferson University Hospital)

Introduction: Prior large, case-control series demonstrate the significant decrease in surgical site infection (SSI) with the administration of vancomycin powder in the wound bed. While infrequent, most SSI reported after vancomycin powder are gram-positive infections. The review the use vancomycin powder to highlight its correlation with gram negative infections, anearobic bacteria, and culture-negative seromas.

Methods: A single institution, electronic database search was conducted for all spinal surgery patients who had received prophylactic crystalline vancomycin powder in the wound bed from January 2011 until June 2013. Patients with a prior history of wound infection, intrathecal pumps, or spinal stimulators were excluded from the study.

Results: 981 consecutive patients (494 male, 487 female, mean age 59.4 years, range 16-95 years) were identified from January 2011 to June 2013. The average dose of vancomycin powder was 1.13 grams. 66 patients $(6.71 \%)$ were diagnosed with a SSI. 51 patients had positive wound cultures (5.2\%). In 15 patients, no organisms were obtained by 72 hours of culturing. The most common organism cultured intraoperatively was staphylococcus aureus (table 2). A number of gram-negative infections were encountered such as serratia marcescens, enterobacter aerogenes, bacteroides fragilis, enterobacter cloacae, citrobacter koseri, pseudomonas aeruginosa. 15 of the $51(29.4 \%)$ positive-cultures were polymicrobial. $8(53 \%)$ of these 15 polymicrobial cultures contained three or more distinct organisms.
Conclusions: Prophylactic intraoperative vancomycin use in the wound bed in spinal surgery may increase the incidence of gramnegative or polymicrobial spinal infections. Large, randomized, prospective trials are needed to demonstrate causation.

\section{Upper Extremity SSEP Monitoring in Posterior Thoracic and Lumbar Surgery to Prevent Peripheral Nerve Injury}

Kenneth M Crandall MD (University of Maryland), Justin Slavin MD (University of Maryland Hospital), Akil Patel MD (University of Maryland Hospital), Gary Thomas Schwartzbauer MD, PhD (University of Maryland), David L Schreibman MD (University of Maryland), Bryan B Ferguson REPT CNIM MCSE (Univeristy of Maryland), David M. Ibrahimi MD (University of Maryland School of Medicine), Charles A. Sansur MD MHSc (University of Maryland)

Introduction: Upper extremity peripheral nerve injury is a risk factor of any operation, particularly posterior spine surgery. Somatosenory Evoked Potential (SSEP) monitoring of the upper extremities has been used to monitor nerve function and possibly prevent injury. Two smaller studies report a $4-7 \%$ incidence of SSEP changes in patients positioned prone with their arms forward. We present the largest series of prone spine patients undergoing SSEP monitoring of the upper extremities.

Methods: We retrospectively reviewed patients undergoing posterior thoracic and/or lumbar procedures positioned prone with their arms forward from 2008-2013. All patients had SSEP monitoring of their ulnar nerves. Patients with a decrease in amplitude of $50 \%$ or increase in latency of $10 \%$ were medically optimized and re-positioned in the operating room. New post-operative neurologic symptoms along with other possible contributing factors were evaluated.

Results: Out of 2475 patients, 73 (2.9\%) had changes in their upper extremity SSEPs. After medical optimization and re-positioning, 34 patients (47\%) had an improvement of their SSEPs. Three of these patents developed new neurologic symptoms in the offending limb. The remaining 39 patients (53\%) had no improvement in SSEPs despite repositioning and two patients developed new neurologic findings. All neurologic symptoms resolved within a few days after surgery.

Conclusions: Three percent of patients developed SSEP changes, lower than previous reported. Five patients had new neurologic deficits, including three in the group with SSEP improvement following repositioning. While repositioning didn't change the incidence of new deficit, none of these patients had any lasting neurologic symptoms after a few days. Other studies of peripheral nerve injury secondary to operative positioning have reported many deficits lasting months to years. Therefore, monitoring upper extremity SSEPs in posterior spine surgery patients and repositioning limbs with changes may be beneficial.

\section{Three- and Four- Level Anterior Cervical Discectomy And Fusion Using PEEK Cages And Plate Fixation For The Treatment of Cervical Spondylosis. Report of 78 cases}

Gabriel A. Gonzales-Portillo MD FAANS FACS, Gabriel S. Gonzales-Portillo, Chiara A. Gonzales-Portillo

Introduction: The choice of surgical approach for the treatment of cervical spondylosis remains controversial. We describe our experience doing three and four levels anterior cervical discectomy and fusion using PEEK cages with Plate Fixation.

Methods: A retrospective review of a prospective collected data was analyzed between 2006-2012. Only patients with more than 3 months of follow up were included. Patients undergoing hybrid constructs were excluded. We analyzed 78 patients with a mean follow up of 15 months. 


\section{Meeting Abstracts: AANS/CNS Section on Disorders of the Spine and Peripheral Nerves}

Results: There were 41 females (53\%) and 37 males (47\%). Mean age was 54 and 53 years respectively. Males presented more frequently with myelopathy, myelo/radiculopathy than females $54 \%$ vs. $19 \%$ and this was statistically significant $(\mathrm{p}<0.0001)$. All patients had a PEEK cage placed. In 20 cancellous ileac crest was used to pack the cage, in 40 local bone was utilized, and in 18 local bone with bone extender were used. No BMP was utilized. Semi-constrained titanium plates were used in all patients. Fusion rates were achieved in all patients undergoing ICBG (100\%) and in $97 \%$ using local bone. Pseudoarthrosis was found in two $(1.3 \%)$ patients with 4 level ACDF and local bone as a graft. Early transient dysphagia was seen in $18 \%$ and there was no difference between 3 levels and 4 levels. One patient (1.3\%) presented with late dysphagia (11 months later) due to a retropulsed screw. Another patient had incidental retropulsed screw and pseudoarthrosis (10 months later); thus rate of pseudoarthrosis 3\%. Both patients had 4 level ACDF with local bone. Overall fusion rate of $97 \%$. No statistical difference between ICBG cancellous bone and local bone. Clinical outcome by Odom's criteria was $96 \%$ excellent or good.

Conclusions: Acceptable fusion rates (97\%) can be obtained in multiple level ACDF using PEEK cages and plate fixation with local bone. The incidence of transient dysphagia is $18 \%$.

\section{The FGFR/MEK/ERK/Brachyury Pathway is Critical for Chordoma Cell Growth and Survival}

Wesley Hsu MD (Wake Forest School of Medicine), Yunping Hu $P h D$

Introduction: The mechanism associated with brachyury-regulated cell growth in chordoma is poorly understood. Fibroblast growth factor (FGF), a regulator of brachyury expression in normal tissue, may play an important role in chordoma pathophysiology.

Methods: A panel of chordoma cell lines were used to explore the FGF/MEK/ERK/brachyury signaling pathway. Specific inhibitors of each step in this cascade were used to assess the role of this pathway in chordoma cell growth and metastasis. The role of this pathway in chordoma metastasis was assessed by measuring factors associated with epithelial-mesenchymal transition (EMT).

Results: Western blots showed that all chordoma cell lines expressed FGFR2, FGFR3, mitogen-activated protein kinase kinase (MEK), and extracellular signal-regulated kinase (ERK), whereas no cell lines expressed FGFR1 and FGFR4. Results of ELISA indicated that chordoma cells produced FGF2. Neutralization of FGF2 inhibited MEK/ERK phosphorylation, decreased brachyury expression, and induced apoptosis while reducing cell growth. Activation of the FGFR/MEK/ERK/brachyury pathway by FGF2-initiated phosphorylation of FGFR substrate 2 (FRS2)-a (Tyr196) prevented apoptosis while promoting cell growth and EMT. Immunofluorescence staining showed that FGF2 promoted the translocation of phosphorylated ERK to the nucleus and increased brachyury expression. The selective inhibition of FGFR, MEK and ERK phosphorylation by PD 173074, PD 0325901 and PD 184352, respectively, decreased brachyury expression, induced apoptosis, and inhibited cell growth and EMT. Moreover, knockdown of brachyury by shRNA reduced FGF2 secretion, inhibited FGFR/MEK/ERK phosphorylation and blocked the effects of FGF2 on cell growth, apoptosis and EMT.

Conclusions: These findings highlight that FGFR/MEK/ERK/ brachyury pathway coordinately regulates chordoma cell growth and survival and may represent a novel chemotherapeutic target for chordoma.

\section{Nasal MRSA Colonization: Impact on Surgical Site} Infection Following Spine Surgery

Vismay Thakkar MBBS (Thomas Jefferson University Hospitals), Saurabh Singhal, George M. Ghobrial MD (Thomas Jefferson
University), Srinivas K. Prasad MD (Thomas Jefferson University Hospital), James S. Harrop MD FACS (Jefferson University Hospital), Alexander R. Vaccaro MD, Caleb Behrend, Ashwini Dayal Sharan MD (Thomas Jefferson University), Jack Jallo MD, PhD, FACS (Thomas Jefferson University Hospital)

Introduction: Previous studies conducted in areas of cardiothoracic, orthopedics and gastrointestinal surgery have identified the importance of nasal Methicillin Resistant Staphylococcus Aureus (MRSA) screening and subsequent decolonization to reduce MRSA Surgical Site Infection (SSI). This is the first study to date correlating nasal MRSA colonization with SSI in spine surgical population.

Methods: We retrospectively reviewed electronic charts of patients from January 2011 to June 2013 who were screened for nasal MRSA colonization within 30 days of a spinal surgery. Patients who tested positive for MRSA were put on contact isolation protocol. None of these patients received topical antibiotics for decolonization of nasal MRSA.

Results: A total of 519 patients were identified; 384 negative (74\%), 110 MSSA+ (21.2\%), 25 (4.8\%) MRSA+. Culture positive SSI was identified in $27(5.2 \%)$ cases and was higher in MRSA positive group than in MRSA- and MSSA+ group (12\% vs. 5.73\% vs. $1.82 \% ; \mathrm{p}=0.01)$. MRSA SSI rate was $0.96 \%(\mathrm{n}=5)$. MRSA SSI developed in $8 \%$ of MRSA+ group as compared to only in $0.61 \%$ of MRSA- group, giving an odds of 14.23 and $p=0.02$. Also, in presence of SSI, MRSA+ group was strongly associated with MRSA positive wound culture $(66.7 \%$ vs. $12.5 \% ; \mathrm{p}<0.0001)$.

Conclusions: Nasal MRSA colonization is a strong predictive factor with 14-fold greater odds of developing MRSA SSI following spine surgery. Preoperative nasal MRSA screening and subsequent decolonization using topical antibiotics may help in decreasing incidence of MRSA SSI after spine surgery. Nasal MRSA+ patients undergoing spinal surgery should be informed regarding their increased risk of developing surgical site infection.

258. Outcomes After Cervical Laminectomy with Instrumented Fusion Versus Expansile Laminoplasty: A Propensity Matched Study of 8587 Patients

Owoicho Adogwa M.D. M.P.H (Duke University Medical Center, Division of Neurosurgery), Shivanand P. Lad MD PhD (Duke University Medical Center), Kevin T. Huang BA (Duke University School of Medicine), Matthew Hazzard MD (Duke University), T. Ryan Owens MD (Duke University Medical Center), Paul Thompson BS (Division of Neurosurgery), Parastou Fatemi, Joseph S. Cheng MD MS (Vanderbilt University Medical Center), Beatrice Ugiliweneza MSPH (University of Louisville), Maxwell Boakye MD

Introduction: Expansile multilevel laminectomies with instrumented fusion and laminoplasty are therapeutic options in cases of multilevel spinal stenosis with myelopathy and ossification of the posterior longitudinal ligament. Controversy remains regarding which operative procedure is better. Here, we assess post-operative re-operation, 90-day complication rates and healthcare resource utilization following both posterior decompressive approaches.

Methods: A dataset was generated from the Thomson Reuters' MarketScan database with the following inclusion criteria: patients age $>18$ undergoing either cervical laminoplasty or laminectomy with instrumented fusion procedure between 2000 and 2009. This database captures patient-level data on clinical utilization, insurance enrollment and links costs with detailed patient, provider and facility information. Propensity score modeling produced a matched cohort balanced on age, gender, comorbidities, and other relevant factors for total health related cost analysis. We examined reoperation, 30- and 90-day post-operative complication rates (renal, cardiac, neurological, DVT/PE, wound infection), length of hospital stay, and healthcare resources utilization. Univariate and multivariate analysis was used to compare outcomes. 


\section{Meeting Abstracts: AANS/CNS Section on Disorders of the Spine and Peripheral Nerves}

Results: In total, 8,587 patients met our inclusion criteria. Twoyear follow-up data was available for 3138 patients, of whom 258 $(8.22 \%)$ had undergone laminoplasty. Patients undergoing laminoplasty had lower complication rate during their index hospitalization (5.81\% vs $9.62 \%$, aOR: $0.556, \mathrm{p}<0.0002)$, and in the 30 -day (aOR: $0.568, \mathrm{p}<0.0002)$ and 90 -day (aOR:0.593, $\mathrm{p}<0.0002)$ periods. Twoyear re-operation rates were similar between both groups $(9.77 \%$ vs. $7.36 \%, \mathrm{P}=0.20)$. However, patients undergoing index laminoplasty had lower costs during their index hospitalization $(\$ 26,129$ vs $\$ 35,483, \mathrm{p}<0.0004)$, and overall during the two-year post-operative period, as seen in unmatched ( $\$ 77,960$ vs $\$ 106,453, \mathrm{p}<0.0006)$, and propensity matched analysis ( $\$ 77,960$ vs $\$ 95,842, \mathrm{p}<0.0006)$.

Conclusions: Our study suggests that cervical laminoplasty compared to posterior cervical decompression and fusion has significantly lower 30- and 90-day complication rates, similar long-term reoperation rates and lower healthcare resource utilization two years post-operation and should be considered a viable alternative.

259. Quantitative Analysis of MRI Signal Change in Patients with Cervical Spondylotic Myelopathy: Results from the Prospective, Multicenter AOSpine North American Study

Aria Nouri BA MD (Toronto Western Hospital), Lindsay Tetreault Bsc (Toronto Western Hospital), Juan Jose Zamorano MD (Neurosurgery Division-Toronto Western Hospital), Kristian Dalzell, Michael G. Fehlings MD PhD FRCSC FACS (Toronto Western Hospital)

Introduction: While the diagnostic utility of MRI in confirming Cervical Spondylotic Myelopathy (CSM) has been well established, its role as a prognostic tool remains equivocal. A significant contributor to this ambiguity has been variation in methodological quantification of signal change (SC) intensities. It is therefore the objective of this study to develop a reproducible as well as novel SC ratio and determine its relationship with surgical outcome.

Methods: Of 278 patients, 97 had MRIs and complete outcome data. T1- and T2-weighted images were reviewed by 3 investigators for hypo- or hyperintense signal intensities. A SC ratio was formulated by measuring SC at the site of suspected pathology against two baseline references $(\mathrm{C} 2 / \mathrm{C} 7)$. ANOVA and chi-square tests were conducted to determine differences in these cord characteristics across three outcome groups: excellent (mJOA $>=17)$, good $(\mathrm{mJOA}=15-16)$ and poor $(\mathrm{mJOA}<15)$.

Results: Sixty-nine percent of patients had hyperintense SC on $\mathrm{T} 2,26 \%$ displayed hypointensity on T1, and combined T1/T2 SC was observed in $25 \%$. The average SC ratio was $1.32 \pm 0.27(0.89$ 2.34). Patients were classified based on mJOA outcome at 1-year follow-up as "excellent" (46.4\%), as "good" (24.7\%) and as "poor" $(28.9 \%)$. A larger percentage of patients in the good group had T1 (41.67\%) and T2 (79.17\%) SC compared to the excellent group (T1: $17.78 \%(\mathrm{p}=0.096)$ and $\mathrm{T} 2: 55.56 \%(\mathrm{p}=0.049))$. A combined $\mathrm{T} 1 /$ T2-SC was more frequent in the good group $(41.67 \%)$ than in the excellent group $(11.90 \%)(\mathrm{p}=0.051)$. Following adjustment for age and baseline mJOA, the SC ratio was significantly different between the excellent (1.24) and good (1.42) group ( $\mathrm{p}=0.015)$.

Conclusions: T1- and T2-signal intensities as well as SC ratio are MRI parameters that could distinguish between patients with excellent outcome from those with good outcome. Thus, our novel quantitative measurement of SC ratio on $\mathrm{T} 2$ represents a new way in which postsurgical outcome may be predicted in CSM patients.

260. The National Neurosurgery Quality and Outcomes Database (N2QOD) Pilot: Analysis of Effectiveness of Surgical Spine Care

Matthew J. McGirt MD, Steven D. Glassman, John J Knightly MD FAANS (Atlantic Neurosurgical Specialists), Praveen V.
Mummaneni MD (University of California San Francisco Spine Center), Gregory Oetting MD, Nicholas Theodore M.D. F.A.C.S. (Barrow Neurosurgical Associates), Oren N. Gottfried MD (Duke University Mecical Center), Saad Khairi (Goodman Campbell Brain and Spine), Meic H. Schmidt MD (University of Utah Health Center), Maxwell Boakye MD, Steven N. Kalkanis MD (Henry Ford Health System), Doron Rabin MD FRCSC (St. Luke's Neurosurgical Associates), Timothy C. Ryken MD MS FACS (Iowa Brain \& Spine Institute), Gregory W. Balturshot BS, MD (Central Ohio Neurological Surgeons, Inc.), James Chadduck , Daniel Robert Fassett MD (Illinois Neurological Institute), Ralph E. Reeder MD, Clinton F. Miller MD (Coastal New Hampshire Neurosurgeons), Thomas B. Briggs MD (Springfield Neurological Institute, LLC), Nicholas C. Bambakidis MD (University Hospitals Case Medical Center), Mark Edwin Shaffrey MD FAANS FACS (University of Virginia Health System), Mark N. Hadley MD FACS (University of Alabama at Birmingham), Dean G. Karahalios MD, Peter D. Angevine MD (Neurological Institute of New York), Michael D. Martin MD (University of Oklahoma Department of Neurosurgery), Matthew G Ewend (CB 7060 POB 2151), Ali Bydon MD, M. Adam Kremer BS, MD (Brain + Spine Center), Langston T. Holly MD (University of California Los Angeles), Jonathan Slotkin MD (Geisinger Health System), Wayel Kaakaji MD, Alexander K. Powers MD (Wake Forest University), Wesley E. Griffitt MD (Aurora BayCare Medical Center), Troy M. Tippett $M D$, Jeffrey W. Cozzens MD (SIU Health Care), Lana D. Christiano MD (Neurological Associates, Inc), Thomas W. Grahm MD, Barton L. Guthrie MD (University of Alabama, Birmingham), J. Frederick Harrington MD (University of New Mexico Health Center), Christopher I. Shaffrey MD FACS (University of Virginia), Eric H. Elowitz MD (Weill Cornell Medical College/Department of Neurological Surgery), Kevin T. Foley MD FACS (SemmesMurphey Neurologic \& Spine Institute), Clarence B. Watridge MD (Semmes Murphy Clinic), Anthony L. Asher MD FACS (Carolina Neurosurgery \& Spine Associates)

Introduction: As publically promoted by all stakeholders, quality registry platforms lie at the center of emerging evidence-driven reform models. In March of 2012, the AANS launched the National Neurosurgery Quality and Outcomes Database (N2QOD) Lumbar pilot with the aim to develop a valid and accurate platform to measure the safety and effectiveness of everyday neurosurgery and spine care.

Methods: A nationwide, web-based, prospective, longitudinal one-year outcomes registry was introduced into spine practices in 25 U.S. states. Using a standardized process of representative sampling, baseline, peri-operative, 3- and 12-month medical record and patient-reported outcomes (PROs) data was entered prospectively. For quality control, automated missing data reports were communicated to sites weekly and $10 \%$ of sites audited.

Results: Seventeen months after launch, 41 practice-groups had contracted and 5329 patients were enrolled, from 188 surgeons, 53 hospitals and 27 U.S. states. Of more than 1,000,000 independent variables, missing data was $<2 \%$. Three-month follow-up was $82 \%$ and 12-month follow-up was $80 \%$. A significant improvement in all PROs was seen at 3 months and 12 months after surgery: back pain(12mo NRS: $3.2 \pm 3.5)$, leg pain(12mo NRS: $4.3 \pm 3.8)$, disability(12mo ODI-gain: $21.2 \pm 20.9)$ and EQ-5D(12mo QALYgain: $0.23 \pm 0.24)$,Figure 1 . However, $11.1 \%$ and $12.1 \%$ of patients did not show any improvement in disability and QALY at 3mo and $12 \mathrm{mo}$, respectively. $80 \%$ of patients returned to work by 3 mo postop with significant variation in time to RTW for all diagnoses, Figure 2. Significant variation was also observed in PROs at individual patient level,Figure 3.

Conclusions: N2QOD, a prospective, nationwide, outcomes registry that spans the continuum of in-patient and outpatient care is feasible with a high degree of data integrity. Lumbar surgery is highly effective in the real-world setting from a patent-centered and population health perspective. However, there is a subset of patients 


\section{Meeting Abstracts: AANS/CNS Section on Disorders of the Spine and Peripheral Nerves}

that do not improve after surgery. Future efforts of N2QOD will focus on identifying this subset of patients by predicting individuallevel outcomes.

261. Functional Outcomes After Lumbar Spine Fusion Between Patients with Spondylolisthesis and those with Degenerative Disc Disease (DDD): A Propensity Matched Prospective, MultiInstitutional Longitudinal Study of 1,741 Patients

Owoicho Adogwa M.D. M.P.H (Duke University Medical Center, Division of Neurosurgery), Paul Thompson BS (Division of Neurosurgery), Parastou Fatemi , Ranjith Babu MS (Duke University School of Medicine), Timothy Darlington, keshov Sharma , Max Ostrinsky Krucoff MD (Duke Univsrsity Hospital), Joel Martin, Joseph S. Cheng MD MS (Vanderbilt University Medical Center), Robert E. Isaacs MD (Duke University Medical Center)

Introduction: Treating degenerative disc disease (DDD) with lumbar surgery remains controversial. However, in patients with Modic changes, moderate-to-severe disc space collapse, no significant affective distress or failure to respond to 1-year of non-operative management, lumbar fusion may be indicated. Here, we assess two-year patient reported outcomes after lumbar spinal surgery between patients with DDD and those with Spondylolisthesis.

Methods: A nationwide, multi-institutional, prospective spine outcomes registry was utilized. Enrollment criteria included available demographic, surgical and clinical outcome data. 1031 (59.21\%) patients with DDD (Group A) and 636 patients with spondylolisthesis (Group B) were included. Patients completed the Oswestry Disability Index (ODI), and the Visual Analog back- and leg pain questionnaires before surgery, then at $3,6,12$, and 24 months after surgery. Propensity score modeling produced a matched cohort balanced on age, gender, comorbidities, and relevant surgical factors.

Results: Cohorts were similar at baseline. Improvements from baseline were observed in both cohorts in all patient reported outcome measures. Patients in Groups A (DDD) showed significant 2-year improvement from baseline in VAS for back pain $(4.10 \pm 2.85$ vs. $8.0 \pm 2.56, \mathrm{p}=0.001)$, leg pain $(4.85 \pm 2.99$ vs. $6.01 \pm 3.07, \mathrm{p}=0.04)$ and ODI $(33.30 \pm 20.48$ vs. $50.00 \pm 16.00, \mathrm{p}=0.002)$. In the propensity matched cohort analysis comparing patient reported outcomes between cohorts, a similar 2-year improvement in VAS for back pain $(\mathrm{A}: 3.90 \pm 2.75, \mathrm{~B}: 3.2 \pm 3.94, \mathrm{p}=0.56)$, leg pain $(\mathrm{A}: 1.16 \pm 3.04$ $\mathrm{B}: 3.98 \pm 2.79, \mathrm{p}=0.001)$ and ODI (A:16.70 $\pm 21.93, \mathrm{~B}: 17.10 \pm 21.13$, $\mathrm{p}=0.69$ ) were observed between both patient groups.

Conclusions: Our study suggests that when indicated, lumbar arthrodesis for symptomatic DDD provides significant improvement in low back pain and functional disability and should be considered a viable treatment option for patients with medically refractory DDD related back pain. Compared to patients undergoing lumbar spine fusion for spondylolisthesis, there was no observed difference in the extent of improvement from baseline, in pain and functional disability, two years after index spine surgery.
262. Analysis of Local Sagittal and Spinopelvic Parameters in a Stand-alone ALIF for L5S1 DDD - Minimum 2-year Follow-up

Luiz Pimenta MD (Instituto de Patologia da Coluna), Rubens Jensen PhD (IPC), Luis Marchi MSc (Instituto de Patologia da Coluna), Rodrigo Augusto Amaral MD, Thiago Coutinho MD (IPC), Thabata Bueno Ms (IPC)

Introduction: Sagittal decompensation following long adult lumbar spinal instrumentation and fusion to S1 occurs in high rates, when surgical procedures for degenerative disc disease usually don't aim to restore local lordosis parameters. Postoperative improvement in L5 incidence and slip angle has been correlated with better outcomes. The purpose of this paper is to analyze the contribution of a standalone L5S1 interbody procedure toward the treatment of degenerative disc disease and study resultant local sagittal parameters.

Methods: A prospective, non-randomized, single center study with up to three-year follow-up. Minimum follow-up was 24 months. 28 patients, mean age 50.1 y/o (31-82, range), mean BMI 26.1. These parameters were measured on lateral lumbar radiographs in standing position: L1S1 and L4L5 lordosis, and sacral slope (SS). Through a mini-ALIF procedure, L5S1 stand-alone interbody fusion was done with a lordotic cage with three looking screws.

Results: No intraoperative complications occurred. Two cases $(7.1 \%)$ evolved with abdominal seroma in the incision. Average surgical duration was 120 minutes and average blood loss, $130 \mathrm{cc}$. Two patients evolved with retroperitoneal hematoma and underwent drainage. Mean preop VAS back score changed from 71 to $34 \mathrm{~mm}$ at 12 -month evaluation $(\mathrm{p}<0.002)$. Mean preop ODI score changed from 45.3 to $29 \mathrm{~mm}$ at 12 -month evaluation ( $<<0.01$ ). Focal lordosis at L5-S1 improved from average $18.6^{\circ}$ to $26.8^{\circ}$ ( $\mathrm{p}<0.001$ ), focal lordosis at L4-L5 decreased from $24.7^{\circ}$ to $20.7^{\circ}$ ( $\mathrm{p}<0.001$ ). Mean SS improved from $30.3^{\circ}$ at pre-op to $35.3^{\circ}$ ( $\mathrm{p}<0.001$ ), indirectly showing PT decrease, once SS and PT are complimentary measurements.

Conclusions: Clinical symptoms were successfully treated using standalone anterior interbody fusion without posterior decompression and supplementation. Stand-alone mini-ALIF provides a biomechanically stable solution with minimal complications and morbidity. Local sagittal parameters were significantly improved to a more harmonic distal lumbar spine, fact that has been increasing recognized on back pain and adjacent segment degen-eration.

Disclaimer: The Journal of Neurosurgery Publishing Group (JNSPG) acknowledges that the preceding abstracts and poster sessions are published verbatim as submitted and did not go through either the JNSPG's peer-review or editing process. 